\title{
Adaptive Intelligent Welding Manufacturing
}

\author{
An analysis of classical sensing, modeling, and control; modern machine learning; \\ and human-robot collaborative approaches
}

BY Y. M. ZHANG, Q. Y. WANG, AND Y. K. LIU

\begin{abstract}
Optimal design of the welding procedure gives the desired welding results under nominal welding conditions. During manufacturing, where the actual welding manufacturing conditions often deviate from the nominal ones used in the design, applying the designed procedure will produce welding results that are different from the desired ones. Adaption is needed to make corrections and adjust some of the welding parameters from those specified in the design. This is adaptive welding. While human welders can be adaptive to make corrections and adjustments, their performance is limited by their physical constraints and skill level. To be adaptive, automated and robotic welding systems require abilities in sensing the welding process, extracting the needed information from signals from the sensors, predicting the responses of the welding process to the adjustments on welding parameters, and optimizing the adjustments. This results in the application of classical sensing, modeling of process dynamics, and control system design. In many cases, the needed information for the weld quality and process variables of our concern is not easy to extract from the sensor's data. Studies are needed to propose the phenomena to sense and establish the scientific foundation to correlate them to the weld quality or process variables of our concern. Such studies can be labor intensive, and a more automated approach is needed. Analysis suggests that artificial intelligence and machine learning, especially deep learning, can help automate the learning such that the needed intelligence for robotic welding adaptation can be directly and automatically learned from experimental data after the physical phenomena being represented by the experimental data has been appropriately selected to make sure they are fundamentally correlated to that with which we are concerned. Some adaptation abilities may also be learned from skilled human welders. In addition, human-robot collaborative welding may incorporate adaptations from humans with the welding robots. This paper analyzes and identifies the challenges in adaptive robotic welding, reviews efforts devoted to solve these challenges, analyzes the principles and nature of the methods behind these efforts, and introduces modern approaches, including machine learning/deep learning, learning from humans, and human-robot collaboration, to solve these challenges.
\end{abstract}

\section{KEYWORDS}

- Arc Welding • Sensor • Robotic Welding • Sensing • Control

\section{Introduction}

In fusion welding (Ref. 1), the base materials (i.e., materials to be joined) are melted to allow them to mix together, with or without addition of a filler. The resultant welds are formed after the mixed melts are solidified. For solid-state welding such as friction stir welding (FSW) (Ref. 2), the temperature of the base materials is elevated to a level such that the base materials gain the flowability needed for being mixed/joined. Brazing/soldering (Ref. 2) is different as the base materials do not flow/move and are not directly mixed. Flowability of the base materials is thus a basic characteristic of welding, which distinguishes it from brazing/soldering.

The flowability needed for welding is typically gained by elevating the temperature of the base materials using an energy source. The energy source or the way to provide the energy is typically reflected in the name of the process. Arc/ laser welding (Ref. 1) uses an arc/laser as the energy source while friction welding (Ref. 2) uses a mechanical energy. The interaction of the energy source with the base materials produces the heat to elevate their temperature, while in FSW the rotating tool is the carrier of the mechanical energy source that interacts with the base materials.

The requirements on the resultant welds are determined by their intended services. Applying energy into the base materials to produce welds meeting the requirements also produces side effects that adversely affect the ability of the resultant welded structure to meet the service requirements. 


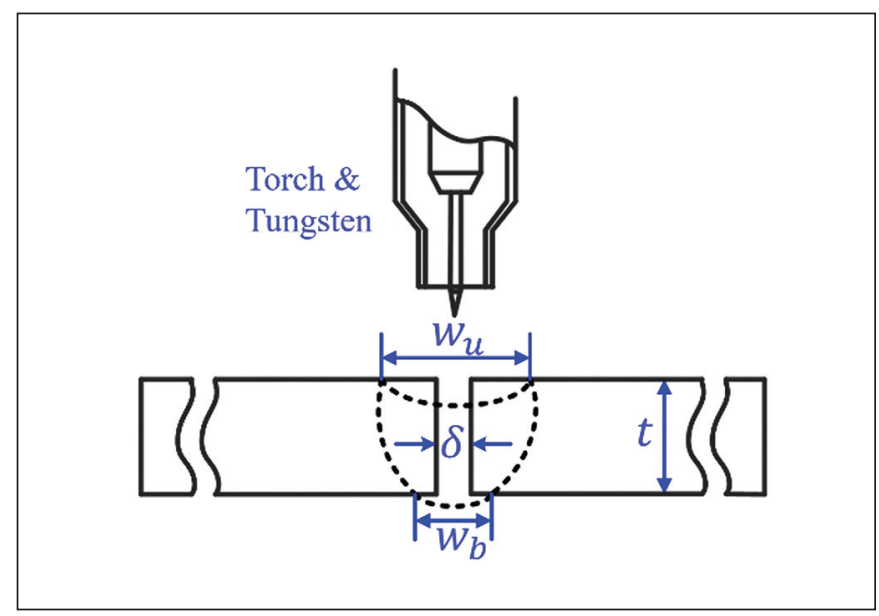

Fig. 1 - Application example to illustrate key issues in adaptive robotic welding.

Some of the adverse side effects are the distortion (Ref. 3) and degradation of the base materials in the heat-affected zone (HAZ) (Ref. 3). Such side effects impose restrictions on how the energy source is applied. An optimal design of the welding procedure (Ref. 4) gives the preferred way to apply the energy source to efficiently produce the desirable welds to meet the service requirements with acceptable side effects.

Implementation of an optimal design requires the ability to deliver the welding procedure as intended. This requires precise application of the energy source as intended. Unfortunately, all optimal designs are based on nominal welding conditions. While efforts need to be taken to minimize the deviation of the actual welding conditions from the nominal conditions on which the optimized design was based, such efforts may be too extensive, costly, or even impossible in many applications. Methods are thus often needed to minimize the deviations of the resultant welds from their nominal ones during the welding manufacturing process. This requires adaptations to make corrections and adjustments during the execution of the designed welding procedure (i.e., during welding manufacturing), resulting in adaptive intelligent welding manufacturing. Efforts addressing the issues and challenges in such adaptations are the focus of review and analysis in this paper.

The following section uses an example to analyze and present the issues and challenges for adaptive intelligent welding manufacturing. A diagram is presented to summarize the principle of an adaptive intelligent welding system and its key elements. The Visual Sensing of the Welding Process section reviews and analyzes the efforts to observe and understand the welding process. The efforts specifically devoted to the monitoring of the joint penetration, one of the most important and challenging targets of adaptive intelligent welding manufacturing, are reviewed and analyzed in the Monitoring of Joint Penetration section. The Modeling and Control section reviews the efforts in modeling of the welding process as a dynamic system and the decisionmaking that determines adjustments of welding parameters based on the dynamic model of the welding process. In the Machine/Deep Learning section, we first introduce the principles of machine learning and then review the use of ma-

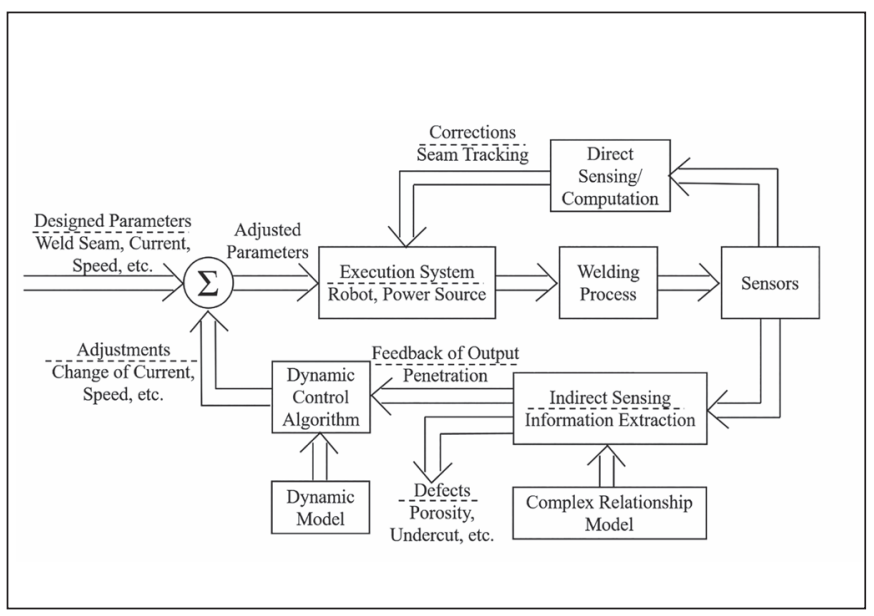

Fig. 2 - Adaptive intelligent robotic welding and its key elements.

chine learning in solving challenges related to adaptive intelligent welding that are difficult to solve using conventional ways. Finally, in the Learning from Human Welders and Human-Robot Collaborative Welding section, we introduce human-robot collaborative welding as an approach to use human adaptation for adaptive intelligent robotic welding and introduce related efforts.

\section{Issues and Challenges}

We use Fig. 1 as a relatively simple application example to illustrate and explain the issues and key elements related to adaptive intelligent robotic welding manufacturing. The joint design of the base materials, which are metals in this example, and the target weld to be produced per the optimal design are given by solid and dashed lines, respectively. The weld is made in a single pass using autogenous gas tungsten arc welding (GTAW) (Ref. 1) without a filler metal. (A second pass may be necessary to gain the needed convexity for the upper surface of the weld but is not of concern here for the illustration example, which is intended to be simple and straightforward.) During welding, the gas tungsten arc (GTA) is applied as the energy/heat source to melt the two members (left and right) of the base metals equally and symmetrically such that the solidified metal forms a weld as the dashed area that can be characterized by the two widths of the weld $\left(w_{u}, w_{b}\right)$ for the major requirements on the fusion of the joint.

The design of the optimal welding procedure has specified 1) the nominal/desired position of the tungsten in relation to the joint and its travel speed along the weld axis, which is perpendicular to the cross section shown in Fig. 1, and 2) the current of the GTA. The welding procedure should have also specified the composition of the shielding gas, the diameter of the gas nozzle, the geometry of the tip of the tungsten, etc., but they are typically kept constant in the design values.

In this example, the most critical parameter in determining the weld is the heat input (Ref. 3), the energy imposed into the base metals per unit length along the weld axis whose cross section is characterized by the gap $\delta$ between the two 


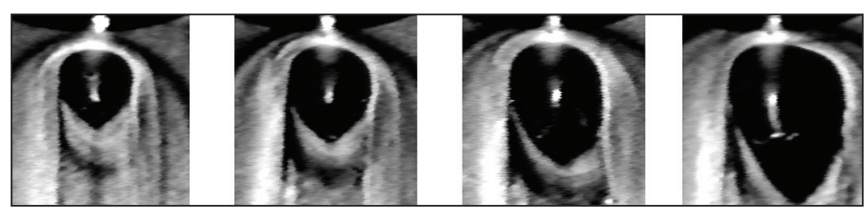

Fig. 3 - Images of the weld pool boundary using a synchronized pulsing illumination laser and camera. Images acquired during studies in Refs. 11-14.

base metals. It equals the power of the arc divided by the travel speed. The power is the product of the current supplied into the arc with the voltage of the arc. When the current and shielding gas are given, the arc voltage is determined by the position of the tungsten above the base metals or the arc length, which is loosely defined as the length of the arc established between the tungsten and the workpiece. In addition to the power, the heat source is also characterized by its distribution. For the same power at a given travel speed, the weld pool is broader and shallower if the distribution is broader. A shorter arc length tends to produce a less broad distribution of the arc energy/heat on the base metals.

Suppose the dashed area is quantified by the upper width and bottom width of the weld. This welding manufacturing process can thus be considered as a system whose outputs are the two widths, that is, $y(t)=\left(w_{u}(t), w_{b}(t)\right)^{T}$, which is the output column vector where $t$ is the time after the welding starts. Optimal design is to determine the welding parameters, current $i(t)$ and travel speed $v(t)$, and welding manufacturing conditions, such as the tungsten transverse position (should aim at the center of the gap), the gap $\delta$, arc length $l$, etc., to produce the desired output $y^{*}=\left(w_{u}{ }^{*}, w_{b}{ }^{*}\right)^{T}$, which is a given vector constant. However, the nominal conditions in the design may not also be assured without excessive efforts as aforementioned. This implies that some of the welding manufacturing conditions such as the tungsten transverse position, arc length $l$, and gap $\delta$ may not be exactly the same as the nominal ones.

One of the major deviations of the welding manufacturing conditions from the nominal ones is the deviation of the tungsten transverse position in relation to the joint/gap. This deviation is referred to as weld seam error. It is critical because an error in the weld seam will result in a wrong position of heat source application. The resultant weld will not be symmetrical as needed. In the worst case, only one of the base metals is melted while another is not. The two members of the base metals are not joined together. This kind of critical deviation must be corrected. That is, seam tracking ability must be provided as a key adaptation during welding manufacturing. On the other hand, an inaccuracy in the arc length, how high the tungsten tip is above the base metals, affects the distribution of the heat source. The output produced will be affected but the weld may still be symmetrical, and the two members of the base metals can still be joined. The correction for the arc length is thus less critical and may be optional.

Because some of the deviations are not practically correctible, the welding parameters and conditions often cannot be the same as the nominal ones. Therefore, the resultant outputs would be different from the desired ones. To produce the desired outputs, some of the easily adjustable welding parameters, such as the welding current or the trav-

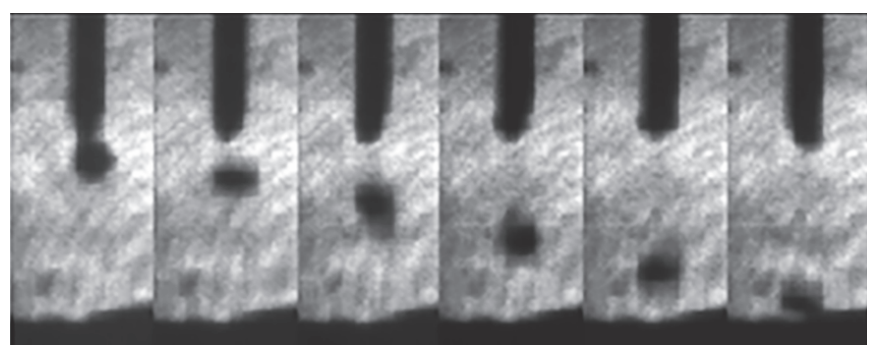

Fig. 4 - Monitoring of metal transfer in GMAW with a backlighting method. Images acquired during the studies for the droplet-oscillation-based active control of the metal transfer process (Refs. 21, 22).

el speed, may be adjusted to compensate for the effect of the uncorrected deviations on the outputs. These real-time adjusted welding parameters form the control variables of the system.

However, how to decide the adjustments of the control variables is not straightforward. The complexity is caused by the dynamics of the system; an adjustment in the control variables would gradually reach its full effect on the outputs. The adjustments on the control variables must be made dynamically, and effective dynamic adjustments not only rely on the availability of the outputs (accurate real-time measurements of the outputs) but also on the understanding of the dynamics of the system (how the outputs dynamically respond to the control variables) and scientific decisionmaking (using the feedback of the outputs and dynamics to decide on the control variables such that the outputs reach the desired values in preferred ways). There are three elements in feedback control: 1) sensing of the feedback, 2) modeling of the system/process dynamics, and 3) control algorithm for decision-making.

Therefore, advanced welding involves at least optimal design of the welding procedure; deviation sensing of the welding manufacturing conditions from the nominal ones to make possible corrections to ensure the best possible execution of the optimal design; feedback control to correct the deviations of the actual produced outputs from the desired ones; process monitoring to find possible defects that are not specified as outputs and also affect the ability to meet the requirements, such as porosities, undercuts, etc.; and post processing and inspection of the resultant welds and welded structures. Optimal design and post processing and inspection are important but are not part of the real-time manufacturing activities. Therefore, they are excluded from the discussion of this paper, which focuses on adaptation of the manufacturing process to the environment (welding/ manufacturing conditions) in intelligent ways.

The major deviation of welding conditions that is sensed and corrected is the weld seam error. Its correction is referred to as the seam tracking (Ref. 3). The upper width of the weld is typically obtained from a view of the liquid weld pool. This and the weld seam may be directly observed from the welding process and we may refer to such a sensing as direct sensing of the welding process. The bottom width of the weld reflects if the base metal has been fully melted in its entire thickness as desired and the degree of this complete joint penetration. If the bottom width is very small and the weld is not perfectly symmetrical as desired, one of 


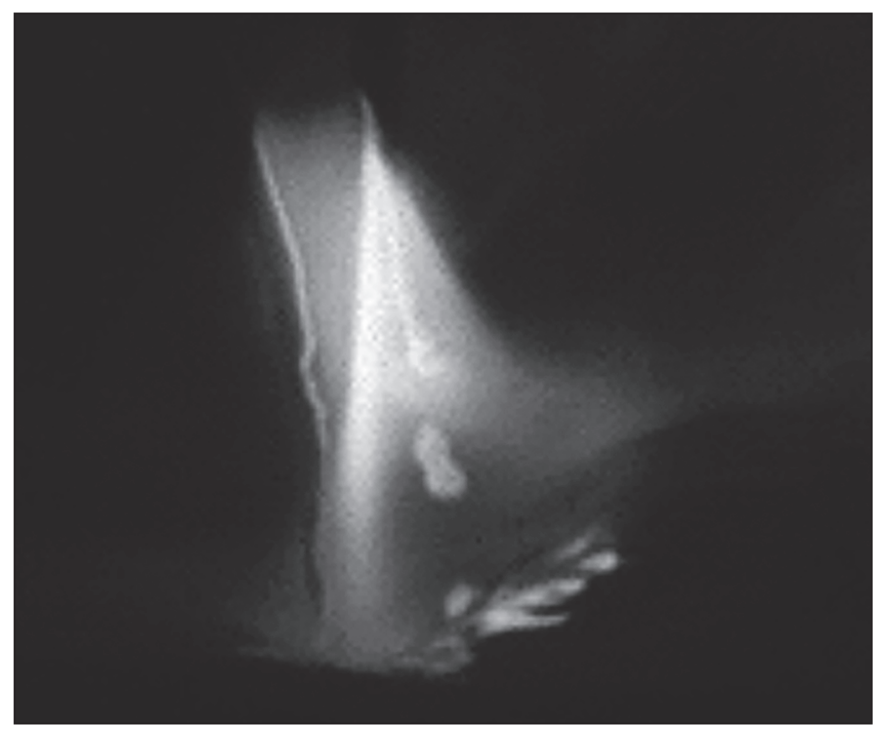

Fig. 5 - Monitoring of metal transfer in GMAW using a highspeed camera. Image acquired during studies for the doubleelectrode GMAW (Refs. 24, 25).

the base metals may have not been fully melted in its entire thickness. In this case, the metal mixing would not occur in the entire thickness of the base metals such that the two members of the base metals are not fully joined together. Therefore, the bottom width is an indicator of the complete joint penetration along the thickness of the base metals. In addition to the complete joint penetration, in certain applications we require the liquid metal does not reach to the opposite side of the base metals. The requirement is typically specified by how deeply the liquid metal reaches underneath the base metal. This type of application is referred to as incomplete joint penetration, and the degree of the incomplete joint penetration is specified by the depth of the penetration (i.e., how deeply the liquid pool has reached underneath the base metal surfaces). The problem for both cases is referred to as the joint penetration. Both of them occur underneath the base metals and are not directly observable. If the output is not directly measurable, we would have to find phenomena that are measurable/observable. This is indirect sensing, and its challenge lies in finding observables that have fundamental correlations with the unobservables (state of the joint penetration) and can be used to fully and accurately determine the unobservables of concern. Defects may be directly or not directly observable.

To summarize, we wish to first point out that we have identified weld seam tracking, joint penetration, and weld defects as major concerns and identified direct and indirect sensing as major types of problems. For direct sensing, the challenges arise primarily from the harsh environment of the welding process - arc radiation, high temperature, smoke, and spatters. For indirect sensing, the challenges lie in 1) if the correlation between the unobservable and observables is fundamental, that is, if the mapping is unique, under what conditions it is unique, etc.; and 2) how to find the mathematical model for the unique mapping such that the measurables are used to effectively determine the unobservables of our concern. All of these form the broad area of research called "real-time sensing of welding process," and the research focuses more on the indirect sensing, that is the extraction of the needed information (unobservable or not directly measured) from the measurables.

Then we wish to point out that the major concerns need to be adaptively controlled to compensate for the effect from the deviations of the manufacturing conditions. To be effective, we also need to model the dynamics of the system. For the welding process, these dynamics are often relatively complex. The welding process is intricate, involving many complex phenomena within the weld pool. The output produced is just part of the welding process with which we are concerned. The dynamics may thus be nonlinear. In addition, there are many parameters that affect the welding process and the output. The control variables are just those that can be easily and effectively adjusted to change the outputs. The uncorrected deviations in the manufacturing conditions also change the dynamic relationship between the control variables and the outputs. The dynamics thus may be uncertain depending on unmeasured manufacturing conditions. Modeling these dynamics mathematically thus may be relatively complex. For such a relatively complex dynamic process, the design of the feedback control algorithm may also not be easy. In addition to the sensing, this paper will survey efforts in modeling and control algorithm design for complex dynamic welding systems.

Finally, we wish to point out that all these research areas are related to intelligent adaptation using machines as shown in Fig. 2. Many adaptations being studied for use by machines may also be provided by human welders who seamlessly integrate abilities of sensing, information extraction, modeling, and decision-making. However, humans are not precise in execution. Human-robot collaboration can be used to combine the adaptation abilities of humans with the precise execution and physical strength of machines/robots to provide another way of intelligent adaptive welding manufacturing. It is another area of study surveyed in this paper.

\section{Visual Sensing of the Welding Process}

Despite the specific purposes that may vary from application to application, a general interest persistently exists to see what is happening during welding. We first wish to see what humans can see, and the visual of the welding process is thus often the first to consider. Human welders are capable of observing the welding process to extract the needed information to make appropriate adjustments so that they can adapt to the changes in the welding manufacturing conditions. Human welders can see where the weld seam is despite the arc radiation. Human welders can see the weld pool (boundary) and its variation. Human welders can also see the 3D weld pool surface. However, all these and other information human welders can see are not easily obtained using machines/sensors. Special requirements are needed for sensors to be capable of sensing what is occurring during welding, especially arc and laser welding.

Arc radiation is the first apparent obscurity for sensors to observe the scenes/objects during the arc welding process. The arc is too bright in comparison with other objects of interest, such as the weld pool boundary and the joint groove/ weld seam. A smart method was proposed by Richardson and Gutow to install a camera to observe the weld pool area 

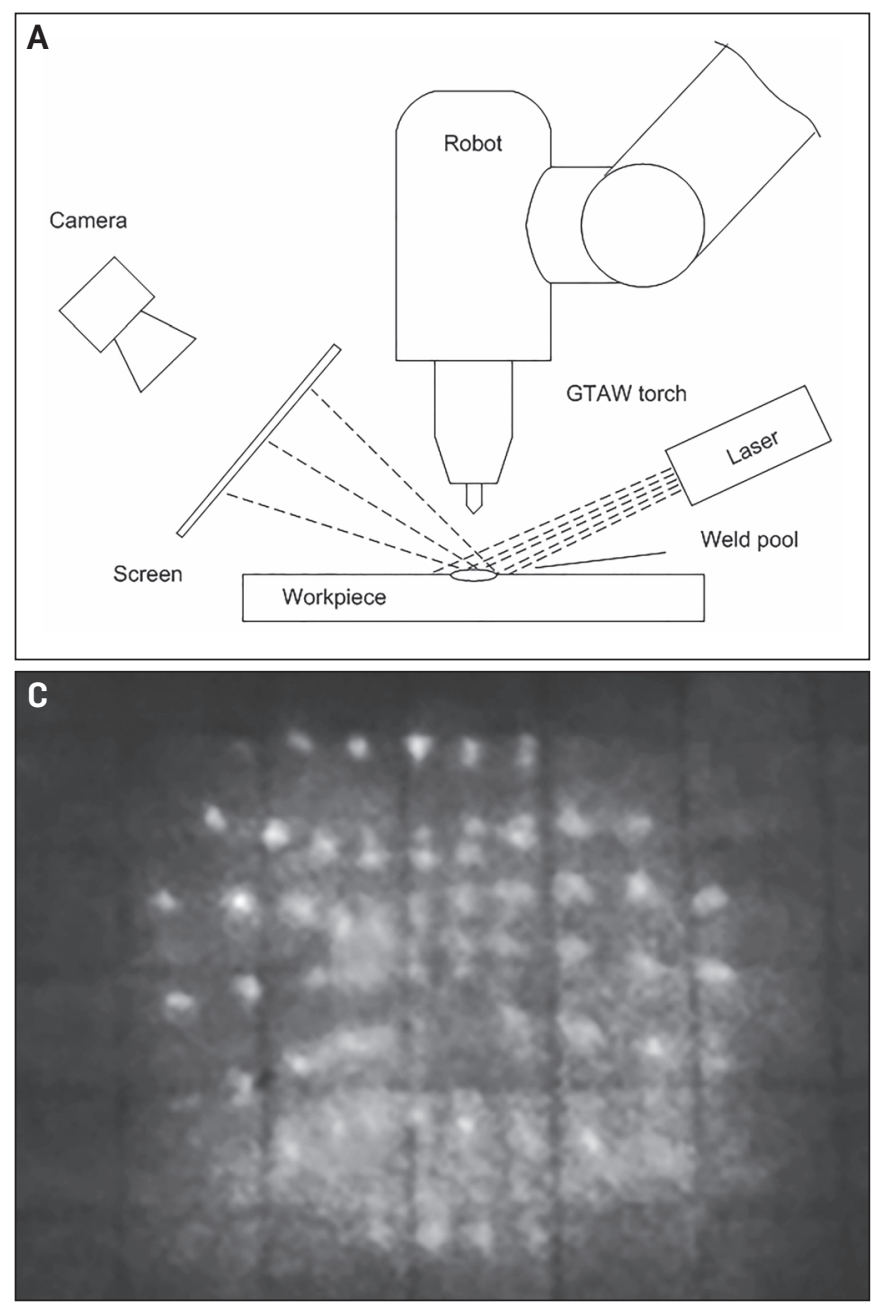

Fig. 6-Measurement of the 3D weld pool surface from its specular reflections to projected laser patterns. Pool surface illustration, images, and results acquired during studies in Refs. 27-29. A - Principle; B - example image using laser stripes as the projected laser pattern; $C$ - example image using a dot matrix as the projected laser pattern acquired during pool oscillation; $D$ - example of calculated weld pool surface.

coaxially from above the tungsten (Ref. 5). This method is referred to as coaxial view of the weld pool, and the tungsten blocks the majority of the arc radiation from reaching the coaxial camera. Clear weld pool images were obtained. To observe from other directions, a direct solution is to use another light source that is even brighter than the arc radiation to illuminate the objects of our interest. This appears to be impossible, but using a laser, which is theoretically single color/wavelength, as the illumination source can make this possible. The energy of the arc radiation spreads in an extremely wide range (Refs. 6,7 ), but the band pass optical filters with the full width-half max of less than $10 \mathrm{~nm}$ are standard commercial off-the-shelf products available for various wavelengths of lasers. And so the energy passing to the imaging sensor of a camera from the object as the reflection of the illumination laser can be much greater than that of the arc radiation. This results in the structured-light method by projecting the laser as a stripe across the joint
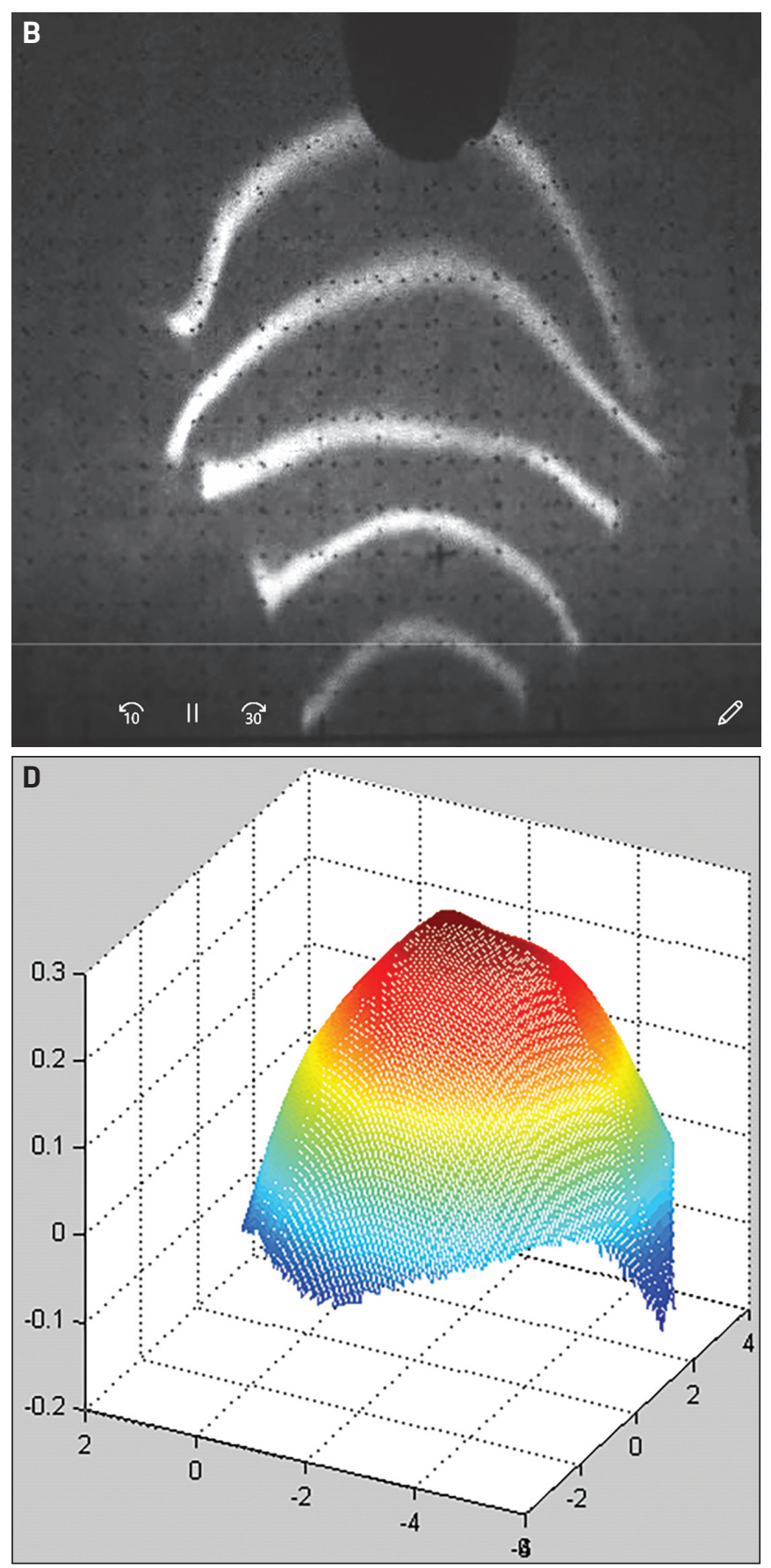

approximately transversely ahead of the weld pool (Ref. 8), which is probably the most effective way to sense the joint groove/gap for seam tracking. To effectively reduce the effect from the arc radiation, the laser stripe is typically projected to the groove with a distance of a few millimeters from the front edge of the weld pool (Ref. 8). To further increase the illumination, the laser is focused, projected as a dot, and scanned across the groove/joint (Ref. 9).

The kind of machine vision methods that use an illumination light source are referred to as active vision (Ref. 10). While the above technologies take advantage of the widespreading characteristic of the arc energy spectral distribution, another method has been based on a different principle - using an illumination light that has much greater 


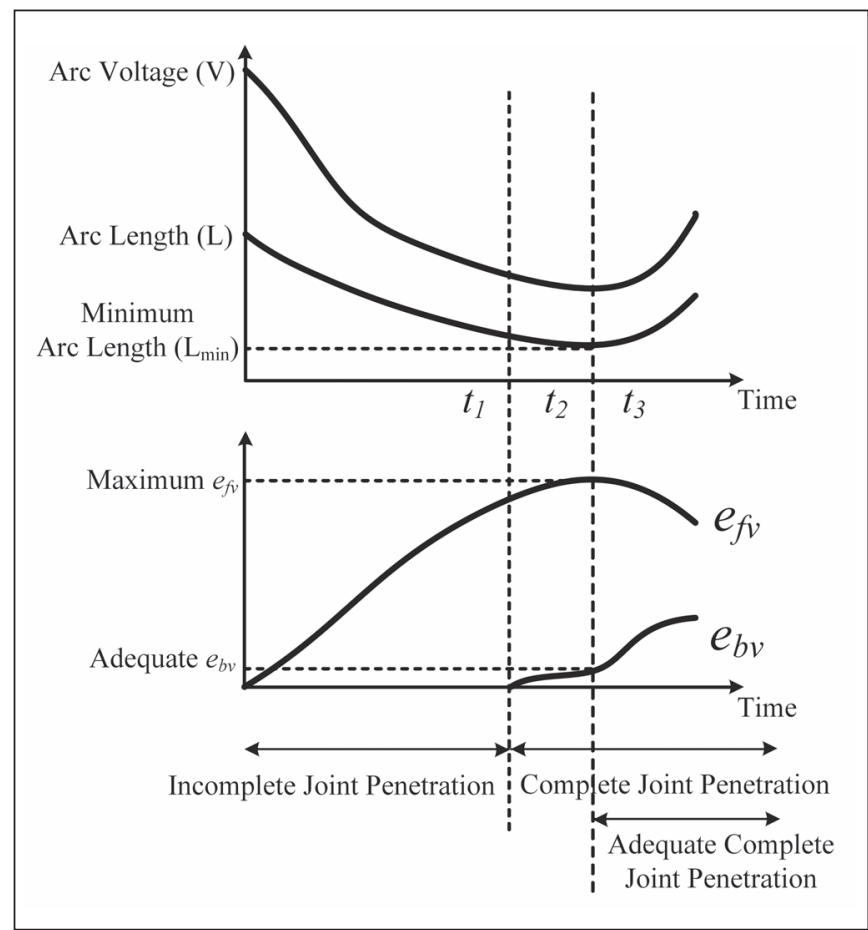

Fig. 7-Arc voltage change during the establishment of the joint penetration. Illustration derived from the studies in Refs. 25 and 26.

power than that of the arc radiation, but just during its pulse (Ref. 11). This method uses a pulsed laser with a duration of nanoseconds. As a result, although the average power of the laser is $7 \mathrm{~mW}$, the peak power during the pulse is $70 \mathrm{~kW}$ (Ref. 11), which is much greater than that of the arc radiation. To sense the reflections from this laser to the onjects, the shutter of the camera is synchronized with the pulse of the laser (Ref. 11). As a result, the arc radiation is completely removed from the image (Ref. 11). The image as shown in Fig. 3 can be obtained (Ref. 11). Using this technology, the weld pool boundary can be clearly observed, where the dark area is the weld pool, which specularly reflects the illumination laser so that the reflection is not collected in the imaging sensor of the camera; the bright area is due to the diffuse reflections of the laser from the solid met- al, which is insensitive to the view angle. Using the technology, the boundary has been clearly monitored, processed, and modeled for process control (Refs. 11-15).

Another important scene in addition to the weld pool boundary is the evolution of the liquid droplet (Ref. 3) pending at and detached from the tip of the welding wire during gas metal arc welding (GMAW) (Ref. 3) and its variants (Ref. 1), including the double-electrode GMAW (Refs. 16, 17). This is referred to as the metal transfer process (Refs. 18, 19), which is critical in understanding the GMAW process. This is because the transfer mode - short-circuiting, globular, or spray transfer - plays a critical role in processing the base metals to produce the welds (Ref. 3). The challenge for monitoring the metal transfer process first lies in the radiation of the arc that roots at the droplet. One method to monitor it is to use a laser to project toward the imaging plane with the droplet/wire in between the laser and imaging plane (Ref. 20). As a result, the droplet blocks the laser, which travels straight. The arc radiation decays cubically such that its incident on the imaging plane is ignorable, but the laser intercepted by the imaging plane keeps its original intensity. A shadow of the droplet and wire is formed on the imaging plane due to the blockage of the laser by the droplet/wire. As a result, clear images of the droplet and wire, as given in Fig. 4, can be clearly acquired to understand the metal transfer process (Refs. 21, 22). This is an active vision method.

The metal transfer can also be monitored using a passive vision method where no illumination/auxiliary light source is used (Ref. 23). In a passive vision method, the camera directly aims at the scenes of interest where both the arc radiation and droplet are present. Using a high-speed camera, the integration time for forming an image frame can be reduced. The brightness associated with the arc radiation can be reduced. The arc radiation can become a suitable illumination source such that the droplet can be seen from the images. Using a neural filter, the arc radiation can be further reduced such that the frame rate needed can be moderate, rather than extremely high, to acquire clear metal transfer images, as examplified in Refs. 24 and 25.

In addition to the weld pool boundary and metal transfer, the 3D weld pool surface is another important scene of interest from which we may gain comprehensive understanding of the welding process. The challenge lies, in addition to
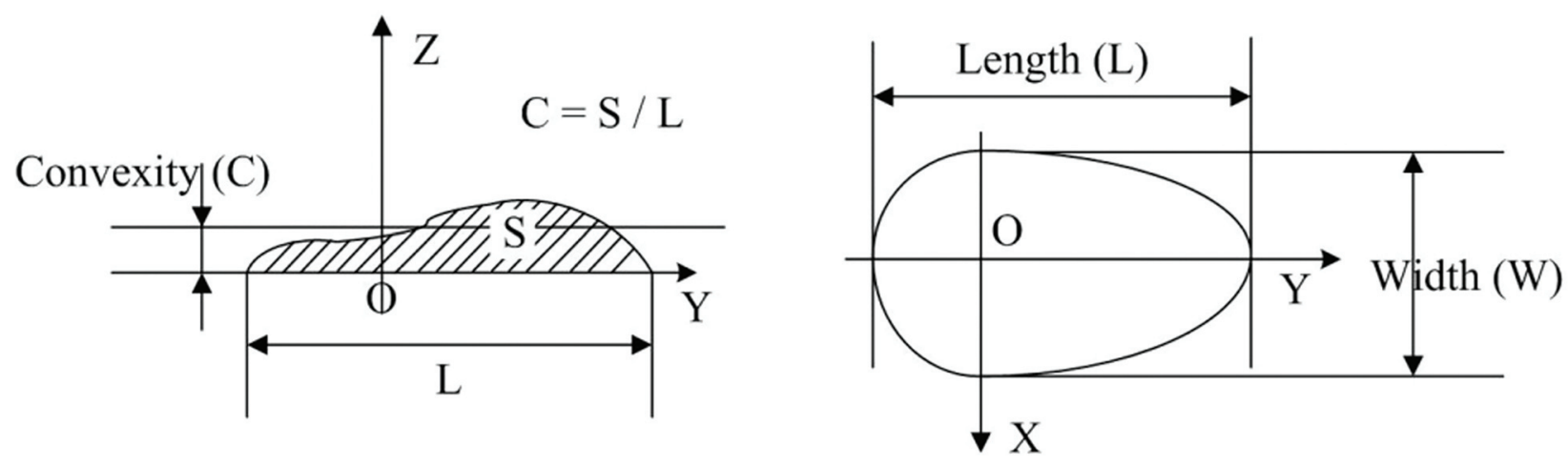

Fig. 8-Characterization of the 3D weld pool surface in GTAW (Ref. 76). 


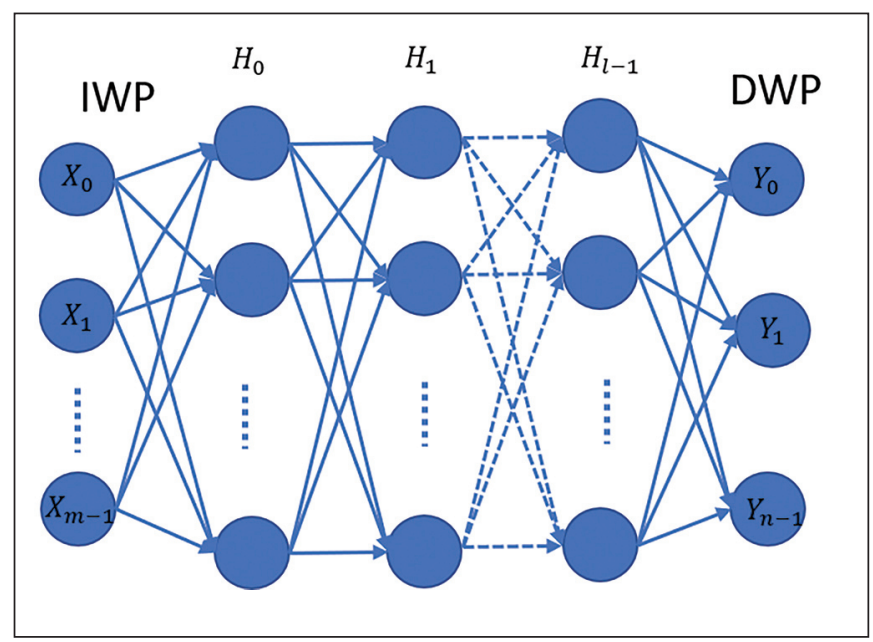

Fig. 9 - Schematic diagram for welding performance using fully connected ANNs with $\mathrm{m}$ indirect welding parameters, $\mathrm{n}$ direct welding parameters, and I hidden layers.

the arc radiation, in the specular nature (Ref. 26) of the surface of liquid metals, such as the weld pool, which disqualifies the structured-light machine vision method that has been used to measure the geometry of an ordinary object whose surface diffusely reflects unidirectionally for collection by a lens from any direction. For specular surfaces, a directionalincident-light-like laser can only be reflected to a given direction following the reflection law. If the lens/camera is not placed in the reflection path, the reflection is not captured/ imaged.

To sense the geometry of the 3D surface, a laser pattern with an array of rays is projected onto the weld pool surface (Refs. 27, 28). Each incident ray is reflected to a particular direction depending on the direction of the incident ray and the slope of the local surface where the incident ray is reflected/ hit. Reflections of the projected rays travel in different directions. When the slope of the surface does not change in an extremely large range, such as from the surface of a GTA weld pool, the directions of the reflections are still within a certain range such that a plane can be placed in their way to intercept the reflections. The intercepted reflections will illuminate the interception plane, and a camera can aim at the interception plane to acquire the image of the reflections. By placing the interception plane at a small distance (Ref. 27), for example 50 $\mathrm{mm}$, the arc radiation significantly delays such that its images on the plane are much less bright than the unidirectional reflection of each incident unidirectional laser ray. Therefore, clear images of the reflection rays can be obtained as shown in Fig. 6. Using the reflection law and constraints due to the smoothness of the weld pool surface, the 3D weld pool surface has been real-time computed (Ref. 28).

The above method to measure the $3 \mathrm{D}$ weld pool surface is theoretically accurate, and the raw image can be very clear. It is also recognizable even if using a dot matrix (such that the intensity of the laser illumination reduces) and under an unfavorable imaging condition of pool oscillation as shown in Fig. 6C. However, it uses a laser pattern and an interception plane (Fig. 6A), reducing its compactness. A passive vision method has also been proposed to measure the 3D weld pool surface (Refs. 30-34). This method views the infrared

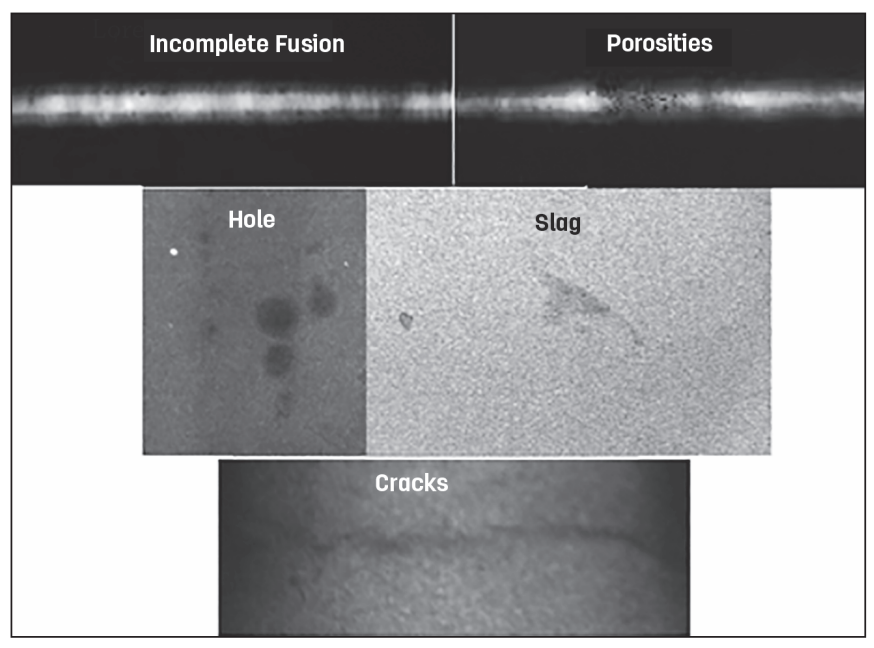

Fig. 10 - The radiographic images of common welding defects from public sources.

radiation, which is determined by the temperature, from the scenes/objects on the weld pool from two directions using the stereo vision principle. This stereo vision method matches the two images, in two cameras, produced by infrared radiation from the same point. If the points matched in two images are sufficient and distribute over the weld pool, the weld pool surface can be fit/computed reasonably. However, the weld pool surface itself lacks features, and the matching is typically first found for feature points/scenes such as oxides/slags and then extends from these well-matched feature points to their featureless neighborhood (Ref. 34). The degree of matching reduces when moving away from the well-matched feature points (Ref. 34). In addition, the arc radiation is much stronger than the useful infrared radiation such that points matched in two images are reduced. Therefore, the effectiveness in measuring the 3D weld pool surface should be much less than the previously aforementioned method based on the specular nature of the surface, although the system becomes more compact. In Ref. 34, a biprism was used to reduce the number of cameras to one.

In addition to visuals, the welding process also has many other features that may be used to understand what is occurring during welding manufacturing. Among them are temperature distribution (Refs. 35-37), sound (Refs. 38, 39), arc light radiation (Refs. 40, 41), arc current waveform, arc length/voltage, etc. In particular, in GMAW, the needed current for the arc anode to complete the melting of the wire is reduced/increased as the wire extension (thus the resistance heat imposed on the wire) increases/reduces. Therefore, the wire can scan across the groove such that the wire extension is changed and the resultant current waveform can be monitored to determine the groove for seam tracking (Refs. 42, 43).

\section{Monitoring of Joint Penetration}

Monitoring of joint penetration is done to determine if the weld pool has complete joint penetration in the workpiece (base materials/metals) to distinguish incomplete from complete joint penetration, and to find the depth of 


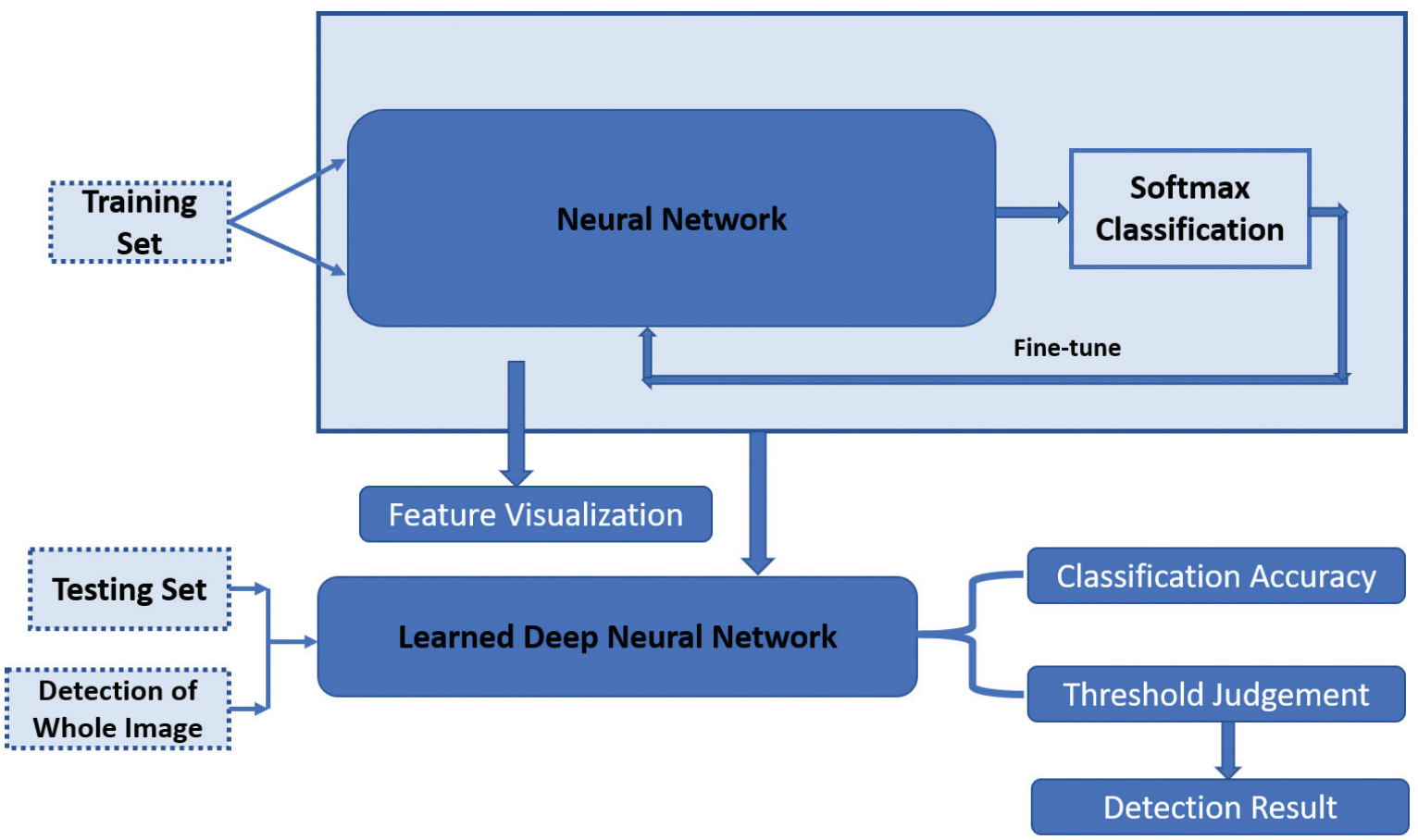

Fig. 11 - Principles of welding defect detection based on CNNs (Ref. 126).

the joint penetration in case of incomplete joint penetration or the bottom width of the weld pool in case of complete joint penetration. In critical applications, such as pipes and pressure vessels where high-pressure, high-temperature gases are transmitted or contained, the unmelted/unjoined interfaces of the two members of the workpiece would cause expulsion. The complete joint penetration must be ensured to meet the service requirements. Because the structures are enclosed after welding, the inspection of the joint penetration from inside becomes difficult. The complete joint penetration thus should be ensured during welding manufacturing. In addition, even if the entire thickness has been penetrated, the bottom width of the weld must be sufficient, in comparison with possible weld pool deviation from the weld seam, which may be caused by seam tracking error and arc blow (Ref. 1), to ensure the interfaces of the base metals are fully melted/joined. Because complete joint penetration is so critical, most of the efforts in this area have been focused on complete joint penetration. The challenge lies in 1) the joint penetration occuring underneath the workpiece and not being directly observable and 2) observables to be measured to determine the joint penetration must uniquely correlate with the joint penetration. To address these challenges, various sensing methods have been proposed.

\section{Pool Oscillation}

Deriving the state of the joint penetration from the oscillation of the weld pool is apparently an indirect sensing method where the observable (from pool oscillation) needs to have a unique correlation with the unobservable (state of the joint penetration) of concern.
Kotecki et al. was among the first to explore the scientific foundation for the correlation being sought (Ref. 44). When a stretched membrane, where the surface tension $\gamma_{0}$ consists of the stretching force, is brought to oscillation by an external force, which is then removed,

$$
\gamma_{0}=3.41\left(a^{2} \rho / t_{0}^{2}\right)
$$

where $a$ is the radius and $\rho$ is the mass per unit area of the membrane and $t_{0}$ is the period of the first harmonic of the oscillation. The oscillation consists of the several harmonics, but the amplitude rapidly reduces as the harmonic order increases. The first harmonic is thus the major component of the oscillation, referred to as the natural oscillation, and its frequency $\left(f_{0}=1 / t_{0}\right)$ is referred to as the natural frequency of the oscillation. When $\gamma_{0}$ is considered a constant, the mass of the membrane $m=\pi a^{2} \rho$ is apparently inversely proportional to the square of the natural frequency $f_{0}$.

The first subsequent follow-up efforts (Refs. 45, 46) in the pool oscillation method 1) assumed this relationship of inverse proportion between the oscillation frequency and the mass/radius/size may also be reasonable for a weld pool, although the weld pool is not a membrane, and 2) focused on experimental verifications of such an inverse proportion or tendency in various conditions. A famous effort was published in the Welding Journal by Richardson and brought the oscillation method wide attention from the welding research community (Ref. 45). In this study, a current pulse was applied to a stationary weld pool and removed to bring the weld pool to an oscillation. Since the arc pressure as an external force imposed on the weld pool is proportional to the square of the current, the removal of the current peak 
changed the external force such that the pool oscillated. The waveform of the arc voltage was then measured as the change of the weld pool surface. The frequency from the analysis was correlated to the diameter of the weld pool in incomplete joint penetration. Another famous study was conducted by Hardt, who proposed a method to determine the back-side bead width by measuring the natural frequency of pool motion when driven by a time-varying arc plasma force (Ref. 46). One should note that, despite many assumptions that appear to be not accurate and scientific, including expending the membrane as a two-dimensional object without a thickness to a three-dimensional weld pool, the tendency between the mass and frequency still holds. However, the accuracy needed for a useful relationship for monitoring and control appears not assured.

The next milestone result that significantly changed the prospective of the pool oscillation method is the finding from Xiao and den Ouden that provides an effective way to distinguish among incomplete joint penetration and complete joint penetration (Ref. 47). They found the oscillation frequency of the pool abruptly reduces after the transition from incomplete to complete joint penetration, from approximately 200 to 50 $\mathrm{Hz}$. This is due to the change in the condition of the bottom surface of the weld pool that was supported by the solid metal in incomplete joint penetration but changed to the surface tension in complete joint penetration. In incomplete joint penetration, the oscillation has never been natural because an external force other than the surface tension has never been removed. For a complete joint penetrated pool, only the "stretching" surface tension is present. The oscillation amplitude is greater, but the frequency is lower. Because of the large difference in the oscillation frequency, the threshold can be easily determined. Along this direction, Andersen and den Ouden developed a synchronous weld pool oscillation method for controlling the weld pool dimensions and state of penetration based on the fact that the weld pool resonates at natural frequencies that are related to its dimensions and state of penetration (Ref. 48).

The way to detect the pool oscillation including the use of signals has also progressed. In early studies, the arc voltage as a measurement of the arc length was analyzed to determine the oscillation (Refs. 45,47 ). It was then found that the arc radiation is more sensitive to the arc length and can be used to better analyze the oscillation (Ref. 49). This is because the arc voltage is the sum of the anode, cathode, and column voltage. While the anode and cathode voltages are constant (Ref. 50) and only the arc column voltage reflects the oscillation, the column voltage only changes with the arc length in a very small range. On the other hand, the arc radiation is primarily due to the arc column whose length is what we need to sense and analyze.

While the previous methods tried to directly measure the oscillation, novel ideas have been proposed to measure the oscillation indirectly but extended from a one-dimensional signal (arc length, arc voltage, arc column) to two dimensional. The information becomes more comprehensive. Shi et al. proposed a novel method to monitor the oscillation by projecting a laser pattern onto the weld pool (Ref. 51). This is the method originally proposed to measure the 3D weld pool surface as illustrated earlier in Fig. 6. Instead of using the reflection law to compute the 3D surface, the movement of the reflection is used to analyze the oscillation of the mirror (sepcular weld pool surface, thus the weld pool) where the projected laser pattern is reflected. This gives amplified signals to analyze the oscillation more easily and accurately. Results showed that weld pool oscillation has the features of incomplete and complete joint penetration and generates two oscillation frequencies.

All previous methods, despite the use of different signals, must wait for a full period of oscillation to analyze the frequency to determine the joint penetration. The monitoring and control are delayed. In Ref. 29, Chen et al. proposed a method that does not actually measure the frequency of the weld pool oscillation. Through studying how the image of the laser dots reflected from the specular weld pool surface in pulsed GTAW change with the dynamic behaviors of a developing weld pool, it was found that the image of the reflected laser dots became less clear and dimmer as the joint penetration increased. Therefore, the brightness of the reflected laser dots can be used to detect the joint penetration instantaneously without waiting for the full period of oscillation.

\section{Ultrasound}

Ultrasound reflects at the interface/discontinuity of materials/material property. By appropriately delivering and collecting ultrasound, this principle may be used to detect the boundaries of the liquid and metal in the weld pool. In Ref. 52, an initial step toward realization of real-time weld pool cross section measurement using reflection ultrasound methods was evaluated. In Refs. 53 and 54, a similar concept was proposed where the joint penetration geometry was detected using a piezoelectric transducer, operating in the pulse-echo mode, to generate shear sound waves that traveled through the base metal to the weld region. The received echoes contained information that could be related to the side-wall joint penetration and thus to the quality of the weld. To overcome the contact requirement of the ultrasonic sensing method, various noncontact ultrasonic sensing methods have been developed, including laser ultrasonic sensing (Refs. 55-57), electromagnetic acoustic transducer (EMAT) ultrasonic sensing (Ref. 58), and laser-EMAT ultrasonic sensing (Ref 59). However, these sensing systems are very expensive and highly complicated, which are not preferable for welding applications.

\section{Infrared}

Infrared sensing is a type of noncontact thermal measurement technique, which has been widely used in various applications and provided important observations to understand abundant welding phenomena (Refs. 60,61). Its use in the monitoring of joint penetration is based on its ability to accurately detect the weld pool boundary due to the work by Chin et al. This is possible because it does not directly measure the temperature, but it measures the intensity of the infrared radiation, which is determined by the temperature and emissivity. When leaving from the center of the weld pool toward the solid metal, the temperature reduces. If the emissivity is constant, the radiation sensed by the infrared camera would gradually reduce. However, at the boundary of the weld pool where 
the solid and liquid metal has the same temperature nominally, the solid metal radiates more strongly because of the greater emissivity. Chin et al. proved that the radiation sensed by the infrared camera indeed has a small increase before it reduces again (Ref. 60). This causes a zero slope in the infrared profile such that the weld pool boundary can be detected without calibration and threshold. In addition to the weld pool boundary, infrared sensors have also been used to monitor weld process parameters including weld bead width, joint penetration depth, and torch position (Ref. 62). More recently, Alfaro and Franco discussed the use of infrared sensors as an indicator for defect detection during the GTAW process using a Kalman filter (Ref. 63). It was shown that infrared signal variations in direct current are related to joint penetration depth, while alternating current portions of the output can be correlated with surface irregularities. However, the correlations between the parameters of concern and the infrared measurements were mostly obtained empirically without a strong support of scientific foundation as the zero slope phenomenon based on which the boundary was detected.

\section{Arc Sensing}

Arc sensing has been used in arc stability control, seam tracking, and joint penetration control (Refs. 64-66). By using the arc itself as a sensor, there is no need for external sensors with their associated costs and concern for reliability in the harsh welding arc environment. A general impression is that the arc signal only contains limited, indirect, and mostly inaccurate information. However, a recent study made arc sensing a promising method to monitor and control the process to ensure complete joint penetration (Refs. 67,68). This method measures the change of the arc voltage and switches the current from peak to the base when the arc voltage tends to increase. This is because when a peak current is applied to establish the joint penetration, the metal melted gradually increases. Due to the thermal expansion, the metal volume increases. Before the bottom of the workpiece is melted, the increased volume of metal can only increase the convexity of the weld pool such that the distance between the weld pool surface to the tungsten decreases. The arc voltage thus gradually decreases because the arc length decreases - Fig. 7. However, after the complete joint penetration is established such that the melted metal may move behind the bottom surface of the workpiece, the arc length will start to reduce more slowly and then increases after more metal moves behind the bottom surface as the volume increases due to thermal expansion. Therefore, an appropriate analysis of the arc voltage that measures the arc length can lead to a reliable detection of the establishment of complete joint penetration, as well as the degree of the complete joint penetration. Since there is no calibration and threshold, the physics foundation is solid, and there is no actual sensor attached, it may be a superior solution that also works in manual welding.

\section{Vision}

The weld pool surface provides important information for understanding welding processes and is the most direct source of information for human welders to obtain feedback. Observing the weld pool surface and measuring its pa- rameters is thus vital to developing intelligent welding machines that can mimic a skilled human welder, who estimates the welding process by visually observing the weld pool and adjusting welding parameters accordingly.

Pioneering work on weld pool observation was conducted by Rokhlin and Guu using radiography (Refs. 69, 70). It was found that the radiation of the received $\mathrm{x}$-ray increases with the depression depth. However, the principle behind this method is to measure the material thickness. For complete joint penetration where the backside pool surface deformation occurs, the pool surface shape will be difficult to extract. Mnich et al. developed a stereovision system for insitu weld pool measurement, where two high-speed cameras were synchronized with a robotic welder to capture images during the time when the arc was off (Ref. 30). By using stereo image processing algorithms, the weld pool shape was rendered in $3 \mathrm{D}$. Zhao et al. used the shape from the shading algorithm to reconstruct the surface from one single weld pool image (Ref. 71). Kovacevic and Zhang proposed a mechanism for observing the GTA weld pool surface shape using a short duration pulsed laser synchronized with a high-speed camera and a frosted glass (Refs. 12, 72). The proposed method can obtain specular reflections from the weld pool under the presence of the bright arc. However, the synchronization of the laser and high-speed shutter requires specific, costly, and sophisticated equipment.

While the laser-camera synchronization can be considered a "hard" way, a "soft" way may be to take advantage of the difference between the arc light and illumination laser. It is known that arc light intensity decreases rapidly with the distance, but the laser does not significantly lose its intensity as distance increases. Saeed and Song studied the structured light method in sensing the GTA weld pool surface (Refs. 73, 74). This approach makes use of a relatively simple idea to facilitate a practical solution to overcome the aformentioned two challenges (bright arc and specular surface): by exploiting the propagation difference between the laser and plasma, the laser rays mirrored from the weld pool surface can be clearly imaged in the presence of bright plasma, thus changing the specular surface from a difficulty to an advantage. More specifically, the reflected laser keeps straight travel without significant loss of intensity over distance, while the radiation of the arc energy decays rapidly over distance. However, the quality of the reflected images is highly sensitive with the shape of the weld pool. Zhang et al. modified the image processing algorithm accordingly, and the $3 \mathrm{D}$ weld pool surface can thus be reconstructed in real time (Ref. 75). The weld pool width, length, and convexity (Fig. 8) were further proven to be an acceptable set to effectively characterize the 3D weld pool surface geometry representing the joint penetration (Ref. 76).

\section{Modeling and Control}

As analyzed earlier, the welding process is dynamic and may also involve any complexity such as nonlinearity. Therefore, the determination on how to adjust the control variables to correct the deviation of the outputs from their desired values is relatively complex and requires an appropriate control algorithm. Availability of the feedback of the output/state is a necessary condition for the adjustments to be effective but is 
not the sufficient condition. The control algorithm must also incorporate the dynamics of the process either during its design or during its on-line implementation. The model predictive control (Ref. 77) explicitly uses a dynamic model of the process being controlled to predict the future process output/ state as a function of the adjustments of the control variables and minimize the difference between the prediction and the desired output/state to optimize the control variables. Other control methods, such as the pole placement, use the dynamic model in the design phase. The following is devoted to providing an overview of the control methods developed for welding processes. Depending on the sensing method used, the control systems can be categorized into pool oscillation-based control, radiography-based control, thermal-based control, and visionbased control. The modeling is part of the efforts in the establishment of these control systems and is not separately reviewed.

Hartman et al. developed a control system that regulated the total heat input to maintain constant fusion zone geometry by monitoring the arc light reflection from the oscillation of the molten metal surface (Ref. 78). Ju et al. proposed a new vibration method: the pulse shielding gas oscillating method (Ref. 79). A control system was constructed by controlling the welding current based on the natural vibration frequency measurements from an arc sensor. Yudodibroto et al. implemented the joint penetration control based on the weld pool oscillation sensing method during the GTAW process with cold filler wire addition (Ref. 80). Guu and Rokhlin utilized realtime radiography for controlling the joint penetration in solidified areas and depressions of the weld pool (Ref. 81). In Ref. 82, Yahia et al. implemented automatic control and inspection of welding defects with an edge detection method of radiographic images based on multilayer perceptron. Song and Hardt developed a closed-loop system to control the weld pool depth using a thermally based depth estimator from point temperatures measured on the backside of the workpiece (Ref. 83). In Ref. 84, a self-organizing fuzzy control method was proposed to obtain a uniform weld pool size in GMAW by regulating the surface temperature at a desired level. Banerjee et al. furthered their earlier finding that the slope of the infrared intensity became zero when the liquid-solid interface of the weld pool was crossed, which might be utilized to control the welding process (Ref. 85).

Compared to the above-mentioned control methods, a vision-based control method is considered more direct and prominent as an emulation of the estimation and decisionmaking process of the human welder. Extensive research has been conducted to explore the possibility to control the weld pool to some extent. Vorman and Brandt used a line scanner to detect the weld pool region and control the weld pool width (Ref. 86). Ohshima et al. proposed a pool width control in pulsed GMAW by observing the weld pool using a chargedcouple device camera in low current intervals of the pulsed current (Ref. 87). Pietrzak and Packer developed a weld pool width control system that uses a miniature camera mounted in a modified coaxial viewing torch to view the weld pool (Ref. 88). Song and Hardt controlled the depth and width of the weld pool using top-side and back-side sensors (Ref. 89). Zhang and Kovacevic proposed adaptive predictive and neurofuzzy model-based control algorithms to control the front-side weld pool width and back-side bead width (Refs. 90, 91). Chen et al. proposed a self-learning fuzzy neural network controller to simultaneously control the weld pool length and width (Ref. 92). Zhao et al. developed a control system to control the backside bead width and front-side reinforcement (Ref. 93). Tsai et al. proposed an automatic pulsed GTAW pipe welding system with fuzzy control technique to control the width of the pool (Ref. 94). Kong and Chen (Ref. 95) and Chen et al. (Ref. 96) controlled the penetration of $\mathrm{Al}$ alloy welding through passive vision of the weld seam and weld pool. Liu et al. controlled the weld penetration and 3D weld pool surface by utilizing the 3D weld pool sensing system and characterization of the weld pool surface (Refs. 97-99). In a separate effort, the welding process was controlled by either directly mimicking the human welder behavior (Refs. 100-103), or by fusing the human knowledge and machine intelligence (Refs. 104-107). Specifically, in Ref. 105, controlling human arm movement was realized for a virtualized welding system with a human in the control loop.

Most of the above efforts are considered empirical methods where the model is drawn from the data obtained from welding experiments. Researchers have also established numerical models to analyze the correlation between the weld pool and arc/heat source, as described in Refs. 108 and 109 for example. These models, in theory, could provide detailed information about the thermal dynamics of the arc and its interactions with the weld pool, which may help us understand the underlined complex correlation between the welding process inputs and the weld pool. However, these numerical models typically require extensive calculations to simulate the complex dynamics, which are not suitable for real-time weld pool monitoring and control applications.

From a modeling/control methodology perspective, the above efforts can also be categorized as linear modeling (Refs. 99, 104), nonlinear thermal modeling (Refs. 83, 108, 109), fuzzy modeling (Refs. 78, 84, 94), shallow neuronetwork modeling (Refs. 82, 96), and neuro-fuzzy modeling (Refs. 91, 98, 100-102), etc. Although they could model and control the welding process with certain success, complex dynamics and highly nonlinear phenomena require more sophisticated models to accurately model such behaviors. In the following section, artifical intelligence and machine learning is introduced along with its application in welding process modeling and control.

\section{Machine/Deep Learning - Standardizing the Learning of Complex Relationships Directly from Data}

Referring back to Fig. 2 illustrating the adaptive intelligent robotic welding and its key elements, indirect sensing/ information extraction and a dynamic control algorithm appear to be relatively less straightforward. They both involve decision-making from relatively complex information sources. For the dynamic control algorithm, its inputs and outputs are both well specified and the complexity is primarily from the dynamics; for the information extraction, the outputs of our concern, such as the state of the joint penetration or weld defects, are well specified, but the inputs are not. It is the job of researchers to 1) hypothesize that the data from selected sensors contains sufficient information to derive the outputs of concern, 2) study the subject/ 


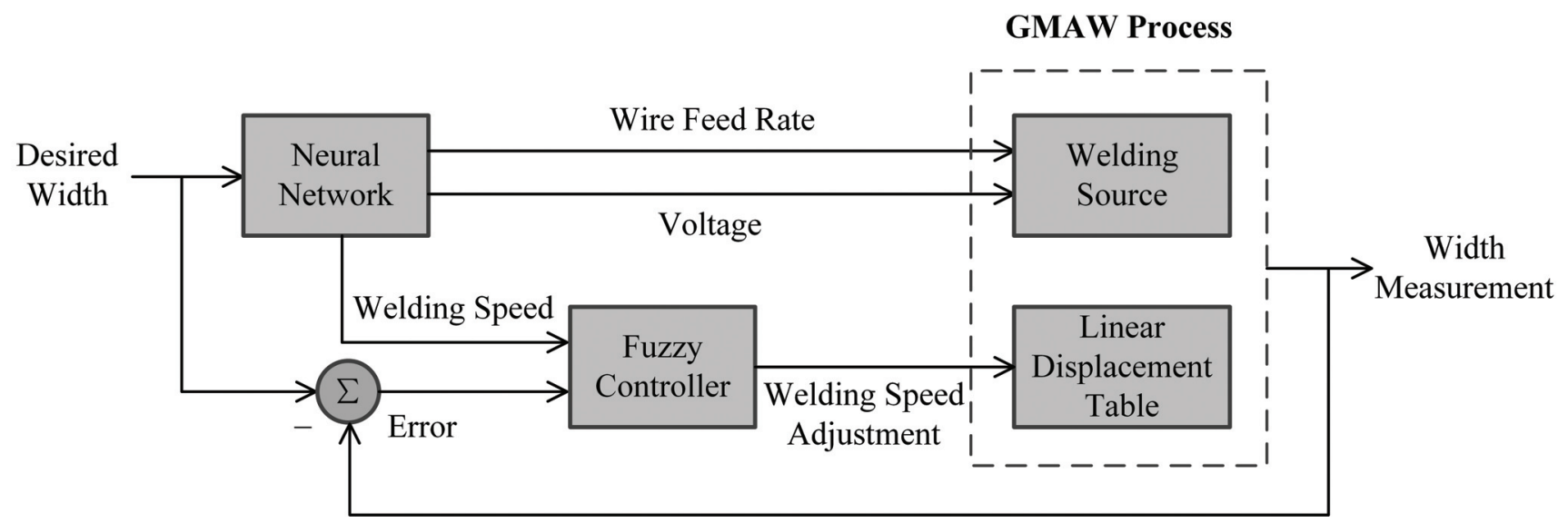

Fig. 12 - Principle of weld bead width control based on ANNs and fuzzy control (Ref. 131).

domain to determine what are the features (useful and efficient representation) of the sufficient but redundant information, 3) propose a model structure to correlate the features to the outputs, and 4) finally fit the model parameters and verify the model. This is the traditional way of information extraction.

It is apparent that this traditional way of information extraction is labor intensive and extremely time consuming, primarily due to step 2 because it is subject/domain specific and requires skilled researchers to perform. The subsequent steps 3 and 4 are also subject specific. This is a human process that is not automated. A possible way to improve the efficiency is to skip the features selection step and directly use the redundant data/information. A more complex, but less subject dependent, model may be used as a class of general model. For each class of general model, a generalfitting algorithm may be developed in advance. Therefore, once the data become available, extracting the needed information becomes an automated process. The efficiency is thus automated/revolutionized. This is the idea of machine learning, especially deep learning (Ref. 110), which uses a class of general model to directly extract the information/ output from the data, which may be redundant. The expense is the need for a large amount of data for the less efficient general model and for extensive computation. The latter has been well addressed by increased/cheap computation power. Collecting/generating the needed large amount of data becomes the only major task for the researchers.

\section{Machine Learning}

In 1959, the concept of "machine learning" was first proposed by Arthur Samuel in his study about artificial players of the game of checkers: programming computers to learn from experience should eventually eliminate the need for much of this detailed programming effort (Ref. 111). Then, Tom M. Mitchell presented a more detailed definition widely accepted by academics: a computer program is said to learn from experience $\mathrm{E}$ with respect to some class of tasks $\mathrm{T}$ and performance measure $\mathrm{P}$ if its performance at tasks in $\mathrm{T}$, as measured by $\mathrm{P}$, improves with experience $\mathrm{E}$ (Ref. 112). The core idea of machine learning is to improve the performance of a task by learning from experience/data. In the last 20 years, with the availability of large-volume data and strong support from computing hardware, especially the highly effective matrix computing in graphics processing units, machine learning has developed rapidly and become a subdiscipline gradually. The architecture of machine learning models become more complex and their functions are more powerful and play an important role in computer vision, natural language processing, decision-making, etc. Compared with traditional methods, machine-learning-based methods have several advantages: 1) Diverse data processing ability: machine learning has the ability to handle multi-dimension and multi-variety data such as images, speech, text, point clouds, graphics etc. 2) Shorter development time: some artificial work in traditional methods can be done by machine learning methods themselves, such as feature selection and model structure optimization. This reduces development time and increases the development efficiency. 3) The ability to continuously improve: the performance can be continuously improved with available large and complex experience. Based on these principles, machine learning can be classified into supervised learning, unsupervised learning, and reinforcement learning.

\section{Supervised Learning}

Supervised learning distinguishes itself from unsupervised and reinforcement learning by its use of label data to specify the desired model output for its paired model input to tune the model parameters. It is preferred that the model input be raw, but redundant, information without preprocessing for the preferred automated process using a class of general model. The raw data can be preprocessed to reduce the redundancy, which reduces model complexity and data size but at the expense of reducing the level of automation. The set of paired data, label, and model input (with or without preprocessing) is split into training and testing data 
sets. The training set is used to train the supervised learning models while the testing set is used for the evaluation and verification of the effectiveness of the trained model. Some common supervised learning models include decision tree, support vector machine (SVM), neural networks, and Bayesian networks. Simpler models may require more preprocessing for the data while relatively complex ones require less.

\section{Artificial Neural Network (ANN)}

In an ANN, a neuro is a nonlinear processing unit/cell whose output is a standardized nonlinear function, with a few free parameters that need to be trained, of its input. Neuros are arranged into layers. The input of a neuro is the weighted sum of the outputs from the neuros in its previous layers or of the inputs of the network for the input layer. The outputs from the neuros in the output layer are the network outputs. Training an ANN is to fit the weights in each weighted sum and the parameters in each neuro. The idea of ANN is to use the aforementioned class of standardized ways of network construction with standardized neuros. The standardization reduces efforts to construct the model structure and helps automate the model fitting.

\section{Deep Learning}

Because of the standardization, the ability to approximate a nonlinear relationship is less effective than a specific model structure. The effectiveness can be increased by increasing layers and neuros. Additional standardized processing functions can also be added, such as the convolution for images and regression for outputs from the last layer neuros. The requirement on the input of the network is reduced such that raw data with redundant information, such as images, can be directly used as network inputs to help automate the modeling process. An ANN is extended with a more complex network to learn more deeply. However, the training becomes more challenging and computation intensive in addition to the increase in the size of needed data. With the recent progress in training methods and algorithms (Ref. 110), such a complex, extended network can be trained using standard algorithms. Deep learning has thus recently gained many successful applications (Ref. 110), including those in welding as reviewed below.

\section{Welding and Machine Learning}

Welding is a chemicophysical process where complex mechanics-heat-metallurgy coupled reactions happen locally. Modeling welding processes based on basic physical rules (e.g., numerical simulation) can help us understand the intrinsic nature of welding itself. However, this computation process is very complex, and the computation volume is too large to be applied in practical manufacturing in real time. Machine-learning-based methods can decrease the computation time and have been applied successfully in welding process modeling. Deep learning is often needed because of the complexity of the welding process.

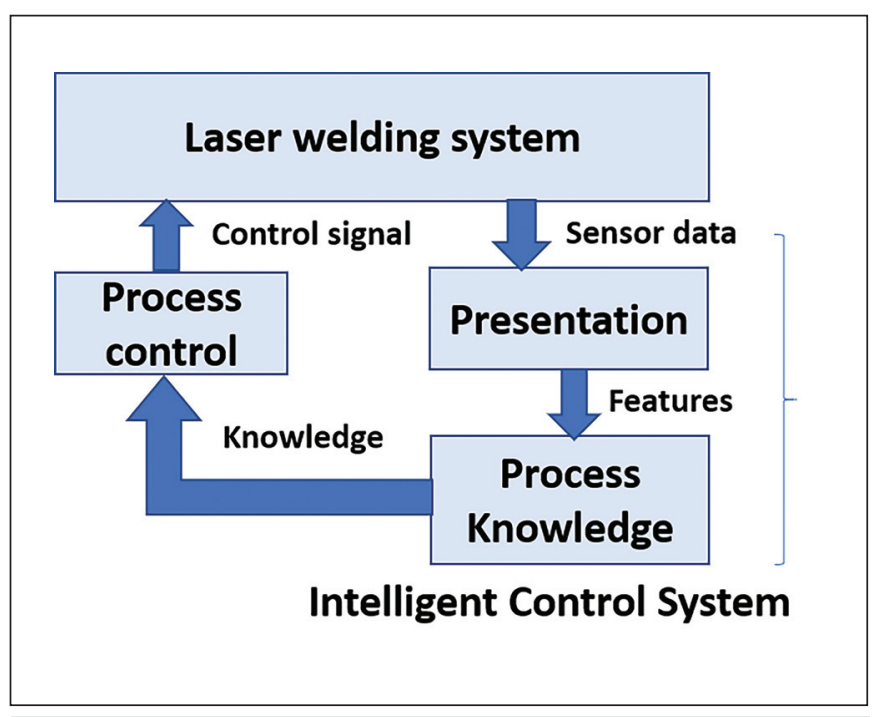

Fig. 13 - Principle of intelligent control of a laser welding system based on auto-encoder and reinforcement learning (Ref. 132).

\section{Welding Performance Prediction}

Predicting the performance of the welded workpiece can help to optimize the welding process and decrease the cost for welding parameters design. The model reported in Ref. 116 is a nonlinear multi-input multi-output mapping model for which machine learning is a feasible tool. This investigative work started in 1990 when Anderson et al. presented the framework and principles for weld performance prediction using ANNs (Ref. 113). The general model architecture of the fully connected ANNs for welding performance is shown in Fig. 9, mapping $m$ indirect welding parameters (IWPs) to $n$ direct welding parameters (DWPs) via $l$ hidden layers. The IWPs are usually the welding parameters applied depending on the specific welding processes, and the DWPs are some characteristic properties of the welded workpieces, including the geometric profile and mechanical properties of the welded joints. By collecting the IWP-DWP data pairs, the ANNs are trained using backpropagation such that nonlinear regression models are built for predicting the welding performance. A lot of research work has been done using this framework for different IWPs and DWPs in different welding methods, which are summarized in Table 1.

\section{Welding Defect Detection}

Welding defect detection is another area where machine learning is widely used. Mirapeix et al. developed a real-time arc welding defect detection system using principle component analysis (PCA) and ANNs (Ref. 124). The plasma spectra of GTAW is collected and preprocessed using PCA such that the data dimension is reduced, and the reductant information is eliminated. Then principle information reserved is used to train an ANN, which has the nonlinear analysis ability to classify the welding defects. Compared with other information sources, a radiograph usually offers comprehen- 


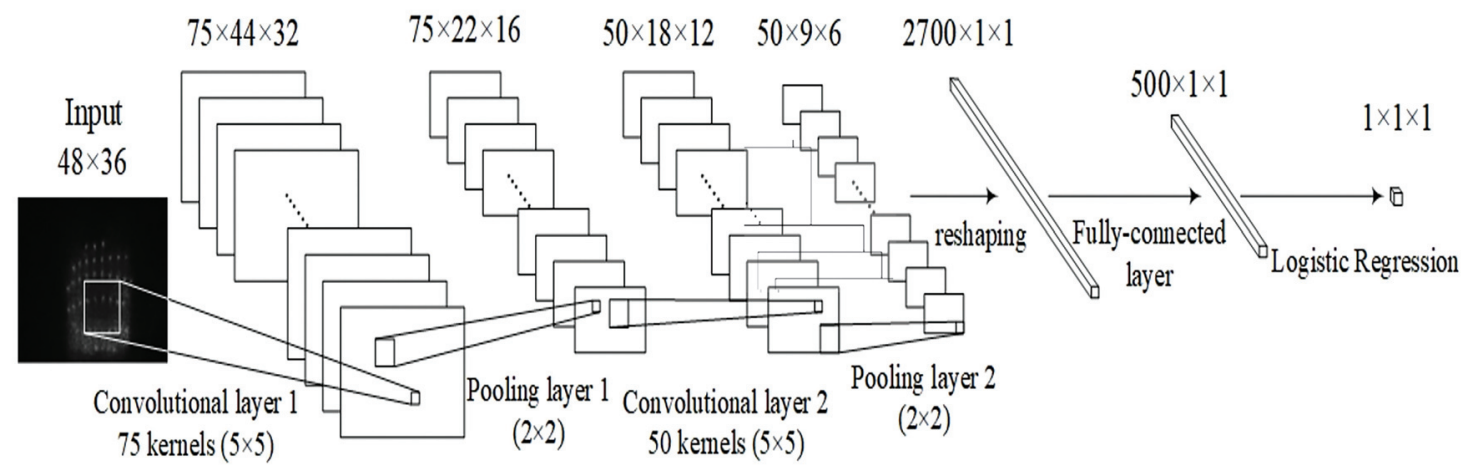

Fig. $14-$ CNN architecture used to predict the weld penetration from a raw image of a reflection from an oscillation weld pool surface (Ref. 134).

sive and reliable information. However, extracting the useful information from the raw images is the foundation to accurately detect the welding defects. Valavanis et al. developed a multiclass welding defect detection system using radiographic images (Ref. 125). First, eight geometric features and 11 texture features are defined and extracted artificially. After analyzing the characterization (nonlinear and high dimensionality), three machine learning methods, including $\mathrm{k}$ nearest neighbor, SVM, and ANNs, are trained to classify six common welding defects, as shown in Fig. 10.

Defining and extracting features artificially depends on the experience of the developers and greatly determines the final accuracy. To address this problem, Hou et al. applied the convolutional neural networks ( $\mathrm{CNNs}$ ) to identify the existence of the welding defects where the features were extracted automatically, as shown in Fig. 11 (Ref. 126). This CNN-based method can learn the feature automatically, reduce the artificial work, and improve efficiency. In CNNs, the number of parameters to be trained is too large such that a lot of data needs to be collected to improve performance and decrease the risk of overfitting. This will greatly increase the cost in time and money to complete many welding experiments. Sassi et al. proposed a transferring learning-based method to solve this problem (Ref. 127). The parameters in CNNs are pretrained using the existing models with good performance in other tasks, and the transferring learning is done with small-volume weld appearance images. This method can decrease the requirement of dataset size without sacrificing performance much. To avoid the shortages due to a single information source, some multi-information sources, including visual information and plasma spectrum, are fused with or without preprocessing to train machine learning models, and the results show that multi-information sources can achieve better and more stable performance (Refs. 128-130).

\section{Welding Process Control}

To achieve high-quality weld joints in practical manufacturing, some machine learning-based control methods have been proposed. Cruz et al. proposed a weld bead width con- trol algorithm based on ANNs and fuzzy control, as shown in Fig. 12 (Ref. 131). The trained ANN will present an optimal three-parameter set including wire feed rate, arc voltage, and welding speed, which are applied in GMAW based on the desired width. Then, a fuzzy controller is applied to control the weld bead width by adjusting welding speed. Reinforcement learning aims to learn a policy for agents interacting with the environment, which is proper for control policy development. Günther et al. developed an intelligent laser welding control system based on auto-encoder and reinforcement learning, as shown in Fig. 13 (Ref. 132). The unsupervised auto-encoder CNN is applied to extract the features from the images of the weld bead. Then, the actorcritic reinforcement learning is used to train a policy for welding quality control. Jin et al. combined the reinforcement learning and proportional-integral-derivative controller to control the width of the weld pool (Ref. 133).

\section{Deep Learning of Joint Penetration Directly from Raw Images}

In the section Monitoring of Joint Penetration, we reviewed an effort that projected a laser pattern onto an oscillating weld pool. Because of the changes in the $3 \mathrm{D}$ shape and in the oscillation amplitude and frequency with the joint penetration, the shape and oscillation of the weld pool surface as the reflection mirror changes with the joint penetration. Since the incident laser pattern is given, the reflection image is only determined by the reflection mirror, thus its shape and oscillation and consequently the joint penetration. This implies that the reflection contains sufficient information to determine the joint penetration However, the oscillation and images are so complex that human review to select/extract the right features accurately is impractical. Deep learning provides a perfect solution to solve this problem.

We used a CNN (Fig. 14) to directly map the reflection image (as exemplified by the image in Fig. 6C) as input to the output that is the area of hot metal on the bottom surface of the workpiece (Ref. 134). This area of the hot metal measures the state of the joint penetration and is obtained from the image taken from the bottom surface of the workpiece as shown 


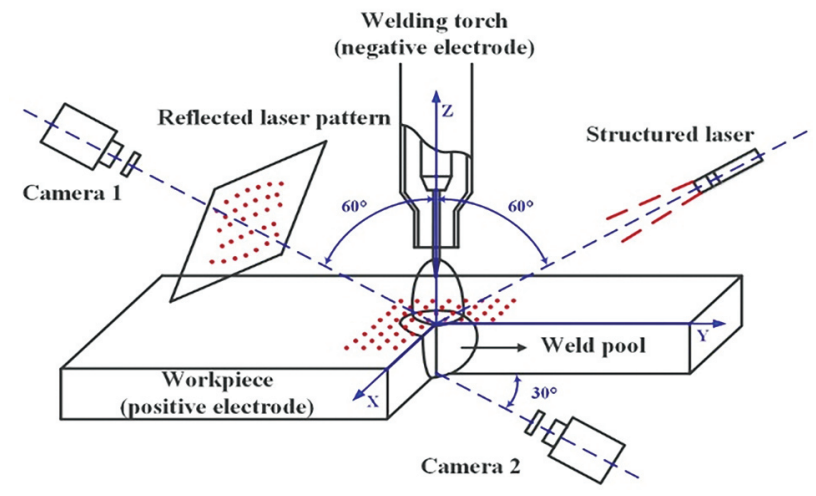

Fig. 15 - Monitoring of reflection image and back-side image (Ref. 134).

in Fig. 15. A reflection image is thus paired with a label. Figure 15 shows how the two cameras monitor the experimental process to obtain the reflection image and the back-side image for the label. Experiments have been conducted to collect 3540 image pairs in various welding conditions. Using data augmentation, we obtained 270,000 training examples, 45,000 validation examples, and 45,000 test examples. The trained CNN gave a final testing accuracy of $90.7 \%$ for classification of joint penetration in six different levels. We also used a voting mech-

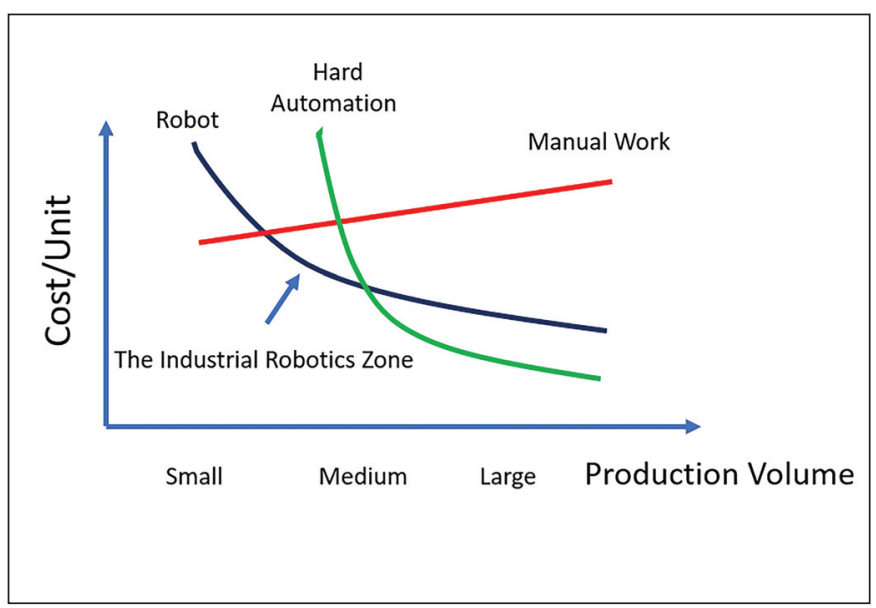

Fig. 16 - Cost performance for different welding performers (Ref. 135).

anism for three neighboring images to increase the classification accuracy to $97.6 \%$.

\section{Learning from Human Welders and Human-Robot Collaborative Welding}

A manufacturing operation may be completed by three kinds of performers: human, robot, and hard automation,

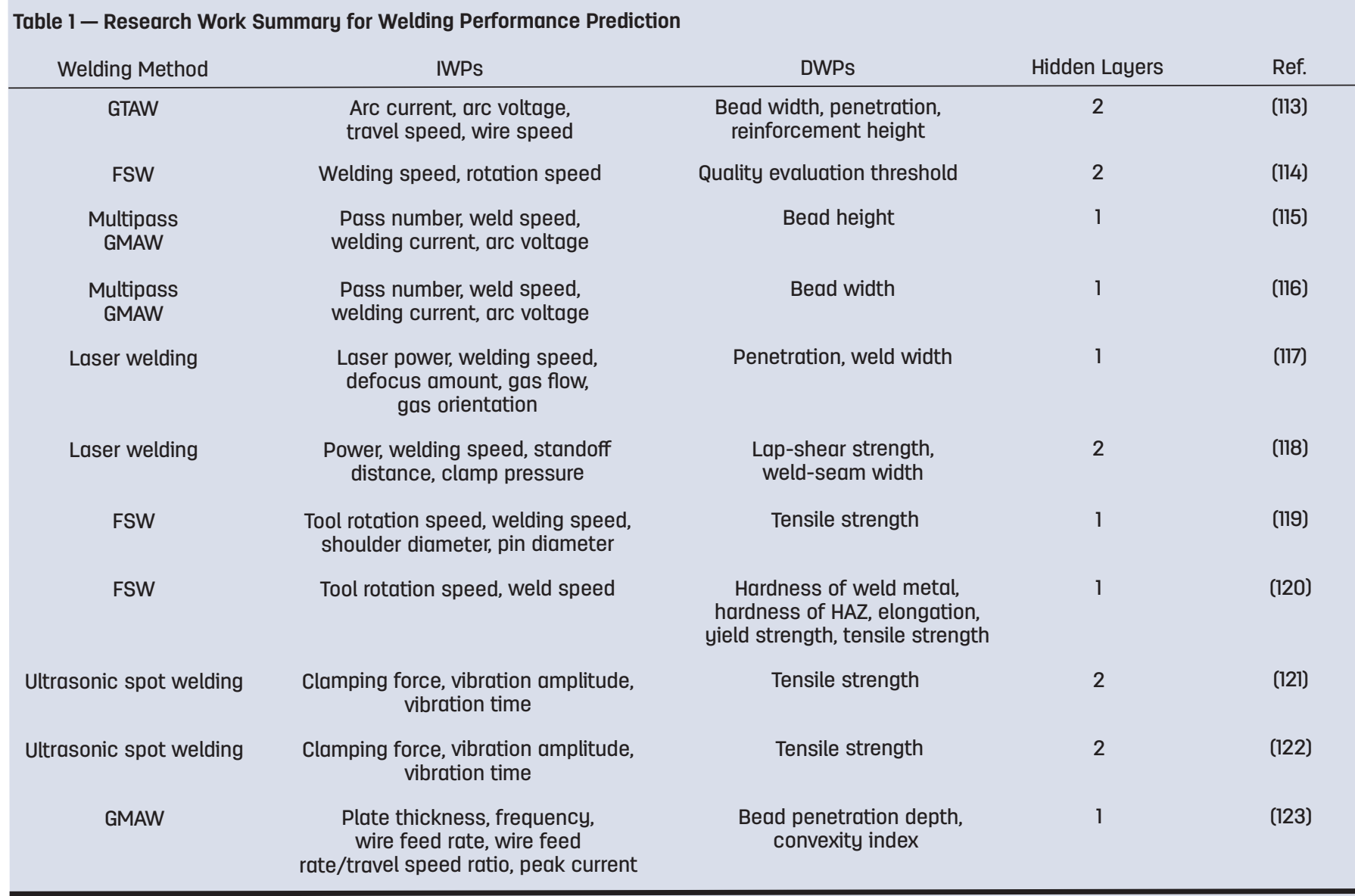




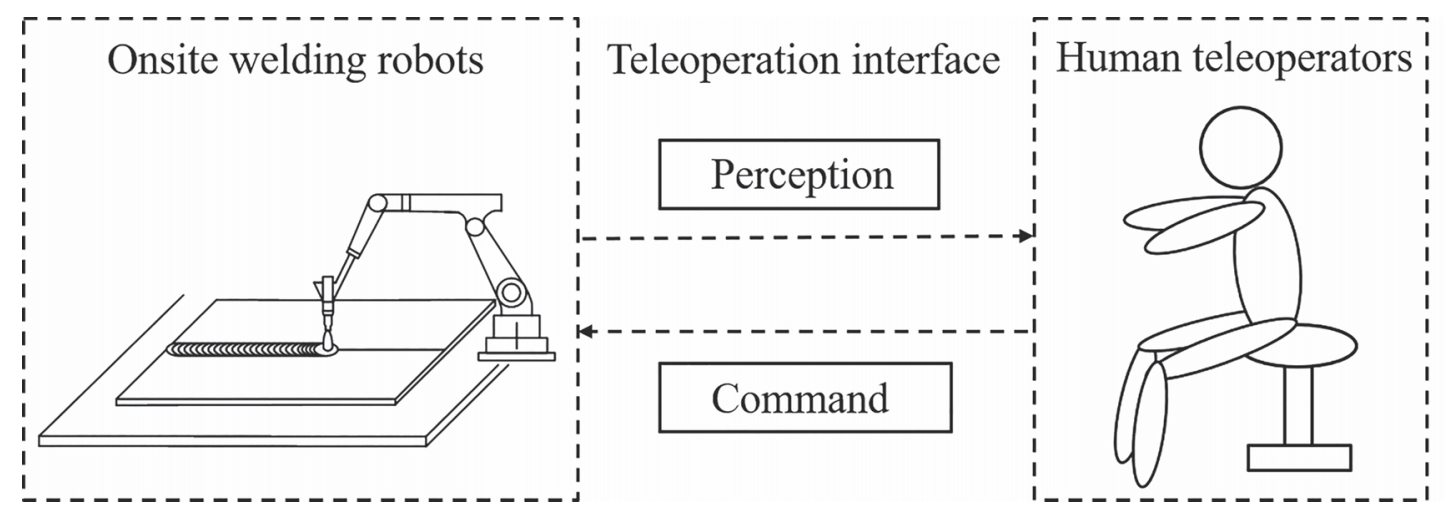

Fig. 17 - General framework of teleoperated welding robots.

where the flexibility is decreasing and the cost per unit is shown in Fig. 16 (Ref. 135). The robots are preferred in small/medium batch manufacturing, which is the mainstream market for current industry. Many welding methods, including resistance spot welding, arc welding, laser welding, and stud welding, have been robotized, and the welding robots account for more than $50 \%$ in the industrial robot market according to the International Federation of Robotics (Ref. 136). Currently, the working principle of the welding robot includes online teaching, offline programming, or a combination of them (Ref. 137). The welding robots execute the preprogrammed action with existing logic and have been successfully commercialized by some traditional robot manufacturers such as ABB, Fanuc, Kuka, and Yaskawa. Methods have been developed to increase the adaptability and flexibility of welding robots.

\section{Learning from Human Welders}

We have demonstrated the challenges in obtaining the needed knowledge to equip welding robots to improve their adaptations. This includes the information extraction and dynamic control algorithm in Fig. 2. However, all these can be performed by human welders relatively easily. This implies that humans have gained the skills through their years of practice for such adaptations. Therefore, we may learn from human welders how they respond to observed phenomena, such as changes in the weld pool surface, to adjust the welding parameters. This requires demonstrations of human welder operations, monitoring of the scene the welder observes to decide the adjustments, and monitoring of the operations of the welder. Most importantly, the sensors for monitoring the scene and welder operation must not be carried out by the human welder so that the welder can operate freely without interference. This requires a special welding and monitoring system. Such a system has been developed at the University of Kentucky to learn the human welder's response to the change in the $3 \mathrm{D}$ surface of the weld pool. The learned model has been integrated into a welding robot so that it can adapt to the welding process and ensure weld penetration (Refs. 138-140).

\section{Teleoperated Welding Robots}

In extreme working environments, robots are the preferred performer for welding tasks because they have higher physical limitations than humans. Due to the current development level of artificial intelligence, there are no fully intelligent welding robots that can perform well in an unstructured extreme environment. Teleoperated welding robots can combine high physical tolerance with the intelligence from humans. The general structure of a teleoperated welding robot is shown in Fig. 17. The welding robots and the human teleoperators are separated spatially and connected via the teleoperation interface. The information flows bidirectionally; that is, human teleoperation commands are transmitted to welding robots, and the welding scenes are sensed and shown to human operators. Some mainstream human-robot interaction interfaces, such as the spacemouse (Ref. 141), haptic controller (Ref. 142), gesture (Ref. 143), and virtual reality (VR) (Ref. 144), have been applied for humans to command the welding robots. Welding scene sensing, reconstruction, and display offer direct perception for human operators, which determines the decision made by human operators. The screen (Refs. 141, 142), VR (Ref. 144), and augmented reality (AR) (Refs. 100, 142) are applied for human operators perceiving the welding scene. From the view of the authors, VR and AR are two highest potential interfaces for welding teleoperation due to the following reasons: 1) The operation styles from VR and AR are the most natural and intuitive. The human operators can adjust the welding torches attached to welding robots like traditional human welders; and 2) the welding scene display styles generate an immersed 3D environment where the human operators can perceive the welding process as if they were onsite.

\section{Human-Robot Collaborative Welding}

Human-robot collaborative welding is a compromised welding style due to the limitation of current full-intelligent welding robots. In a human-robot collaborative welding system, the human and the robot are responsible for the duty they are good at. In such a system, the advantages of the humans and 
robots can be combined. We have developed a human-robot collaborative welding system with a common welding method, weaving GTAW, conducted to verify its effectiveness (Ref. 145). In this system, the robot performs weaving welding with automatic seam tracking based on arc sensors. The human teleoperates the robot movement along the seam via a virtual welding environment generated by a commercial virtual reality system. The intelligent travel speed adjustment from humans could help to decrease the negative effect from environment disturbance. Both the intelligence from humans and movement accuracy from robots are combined so that the welded workpiece has better performance than that from either humans or robots separately.

\section{Robot-Assisted Manual Welding}

In some small-volume and unstructured welding tasks, manual welding is preferred over robots, considering the time and cost investment. However, the global shortage of human welders means skilled welders are not always available for small-volume welding. Some robot-assisted welding systems are developed for novice human welders to achieve high-quality welds. Erden et al. proposed a robot-assisted manual welding system by adding impedance such that the inevitable vibration during novice manual welding is greatly suppressed (Ref. 146). Wang et al. proposed a virtual reality robot-assisted welding system based on human intention recognition (Ref. 147). By modeling and recognizing human intention, the intended operation is extracted and applied to welding processes via robots, and the welding quality is improved.

\section{Conclusion}

Adaptive welding manufacturing is to make real-time corrections and adjust the welding parameters during welding to minimize the effects from not perfectly-controlled welding conditions. This can be considered a feedback control process that first requires sensors to obtain the actual welding conditions for corrections and the feedback of the concerned output from the welding manufacturing process. If the sensor signals are not direct measurements of the feedback, the information extraction sometimes may be complex. Such a complex problem may be solved by using deep learning and learning from human welders. To then make decisions on the adjustments of the welding parameters, we need an understanding of the dynamics of the welding process in response to the changes in the welding parameters/control variables. We also need appropriate ways to make decisions based on the dynamics and feedback. They are the dynamic modeling and control. Therefore, sensing, modeling, and control are the major elements in adaptive welding, and machine learning/deep learning, learning from human welders, and human-robot collaboration are modern ways to provide solutions to these elements.

\section{References}

1. American Welding Society. 2004. Welding Handbook: Welding
Processes, Part 1, Vol. 2, $9^{\text {th }}$ ed. O’Brien, A., ed. Miami, Fla.: American Welding Society.

2. American Welding Society. 2007. Welding Handbook: Welding Processes, Part 2, Vol. 3, $9^{\text {th }}$ ed. O’Brien, A., ed. Miami, Fla.: American Welding Society.

3. American Welding Society. 2019. Welding Handbook: Welding and Cutting Science and Technology, Vol. 1, 10 $0^{\text {th }}$ ed. Sinnes, K., ed. Miami, Fla.: American Welding Society.

4. Liao, B., Shi, Y., Cui, Y., Cui, S., Jiang, Z., and Yi, Y. 2018.

Mathematical model for prediction and optimization of weld bead geometry in all-position automatic welding of pipes. Metals 8(10): 756. DOI: $10.3390 /$ met 8100756

5. Richardson, R. W., Gutow, A., Anderson, R. A., and Farson, D. F. 1984. Coaxial arc weld pool viewing for process monitoring and control. Welding Journal 63(3): 43-50.

6. Węglowski, M. S. 2007. Investigation on the arc light spectrum in GTA welding. Journal of Achievements of Materials and Manufacturing Engineering 20(1-2): 519-22.

7. Li, P. J., and Zhang, Y. M. 2000. Analysis of an arc light mechanism and its application in sensing of the GTAW process. Welding Journal 79(9): 252-s to 260-s.

8. Kiddee, P., Fang, Z., and Tan, M. 2016. An automated weld seam tracking system for thick plate using cross mark structured light. The International Journal of Advanced Manufacturing Technology 87: 3589-3603. DOI: 10.1007/s00170-016-8729-7

9. Xu, P., Xu, G., Tang, X., and Yao, S. 2008. A visual seam tracking system for robotic arc welding. The International Journal of Advanced Manufacturing Technology 37: 70-75. DOI: 10.1007/s00170007-0939-6

10. Xiong, J., and Zou, S. 2019. Active vision sensing and feedback control of back penetration for thin sheet aluminum alloy in pulsed MIG suspension welding. Journal of Process Control 77: 8996. DOI: 10.1016/j.jprocont.2019.03.013

11. Kovacevic, R., Zhang, Y. M., and Ruan, S. 1995. Sensing and control of weld pool geometry for automated GTA welding. Journal of Manufacturing Science and Engineering 117(2): 210-222. DOI: 10.1115/1.2803297

12. Kovacevic, R., and Zhang, Y. M. 1997. Real-time image processing for monitoring of free weld pool surface. Journal of Manufacturing Science and Engineering 119(2): 161-169. DOI: 10.1115/ 1.2831091

13. Kovacevic, R., Zhang, Y. M., and Li, L. 1996. Monitoring of weld joint penetrations based on weld pool geometrical appearance. Welding Journal 75(10): 317-s to 329-s.

14. Zhang, Y. M., Kovacevic, R., and Li, L. 1996. Characterization and real-time measurement of geometrical appearance of the weld pool. International Journal of Machine Tools and Manufacture 36(7): 799-816. DOI: 10.1016/0890-6955(95)00083-6

15. Wu, C. S., Wang, L., Ren, W. J., and Zhang, X. Y. 2014. Plasma arc welding: Process, sensing, control and modeling. Journal of Manufacturing Processes 16(1): 74-85. DOI: 10.1016/j.jmapro. 2013.06.004

16. Zhang, Y. M., Jiang, M., and Lu, W. 2004. Double electrodes improve GMAW heat input control. Welding Journal 83(11): 39-41.

17. Li, K. H., Chen, J. S., and Zhang, Y. 2007. Double-electrode GMAW process and control. Welding Journal 86(8): 231-s to 237-s.

18. Kim, Y., and Eagar, T. W. 1993. Metal transfer in pulsed current gas metal arc welding. Welding Journal 72(7): 279-s to 287-s.

19. Kim, Y., and Eagar, T. W. 1993. Analysis of metal transfer in gas metal arc welding. Welding Journal 72(6): 269-s to 275-s.

20. Allemand, C. D., Schoeder, R., Ries, D. E., and Eagar, T. W. 1985. Method of filming metal transfer in welding arcs. Welding Journal 64(1): 45-47.

21. Zhang, Y. M., and Li, P. J. 2001. Modified active control of metal transfer and pulsed GMAW of titanium. Welding Journal 80(2): 54-s to 61-s.

22. Zhang, Y. M., Liguo, E., and Kovacevic, R. 1998. Active met- 
al transfer control by monitoring excited droplet oscillation. Welding Journal 77(9): 388-s to 395-s.

23. Teixeira, G. S., and Mazzaferro, J. A. E. 2019. GMA welding metal transfer mode study by high-speed imaging and electrical signal acquisition. Journal of the Brazilian Society of Mechanical Sciences and Engineering 41: 315. DOI: 10.1007/s40430-019-1814-8

24. Lu, Y., Chen, S., Shi, Y., Li, X., Chen, J., Kvidahl, L., et al. 2014. Double-electrode arc welding process: Principle, variants, control and developments. Journal of Manufacturing Processes 16(1): 93-108. DOI: 10.1016/j.jmapro.2013.08.003

25. Li, K., and Zhang, Y. M. 2007. Metal transfer in doubleelectrode gas metal arc welding. Journal of Manufacturing Science and Engineering 129(6): 991-999. DOI: 10.1115/1.2769729

26. Ready, J. F. 1976. Change of reflectivity of metallic surfaces during irradiation by CO2-TEA laser pulses. IEEE Journal of Quantum Electronics 12(2): 137-142. DOI: 10.1109/JQE.1976.1069106

27. Zhang, Y. M, Song, H. S., and Saeed, G. 2006. Observation of a dynamic specular weld pool surface. Measurement Science and Technology 17(6): L9-L12. DOI: 10.1088/0957-0233/17/6/L02

28. Zhang, W., Wang, X., and Zhang, Y. 2013. Analytical realtime measurement of a three-dimensional weld pool surface. Measurement Science and Technology 24(11): 115011. DOI: 10.1088/ 0957-0233/24/11/115011

29. Chen, J. S., Chen, J., Zhang, K., Feng, Z., and Zhang, Y. M. 2018. Dynamic reflection behaviors of weld pool surface in pulsed GTAW. Welding Journal 97(6): 191-s to 206-s. DOI: 10.29391/ 2018.97.017

30. Mnich, C., Al-Bayat, F., Debrunner, C., Steele, J., and Vincent, T. 2004. In situ weld pool measurement using stereovision. Proceedings of 2004 Japan - USA Symposium on Flexible Automation, 1-4. Denver, Colo.

31. Neill, A. M., and Steele, J. P. H. 2016. Modeling and simulation of three dimensional weld pool reconstruction by stereo vision. 2016 IEEE International Conference on Advanced Intelligent Mechatronics (AIM), 542-547. Banf, Alberta, Canada: IEEE. DOI: 10.1109/AIM.2016.7576824

32. Lee, D. H., and Kweon, I. 2000. A novel stereo camera system by a biprism. IEEE Transactions on Robotics and Automation 16(5): 528-541. DOI: 10.1109/70.880803

33. Yoo, C. D., and Lee, J. 2000. 3D measurement of weld pool using biprism stereo vision sensor. http://joiningl.kaist.ac.kr/ research/vision.html

34. Liang, Z., Chang, H., Wang, Q., Wang, D., and Zhang, Y. M. 2019. 3D reconstruction of weld pool surface in pulsed GMAW by passive biprism stereo vision. IEEE Robotics and Automation Letters 4(3): 3091-3097. DOI: 10.1109/LRA.2019.2924844

35. Villar, M., Garnier, C., Chabert, F., Nassiet, V., Diez, J. C., Sotelo, A., et al. 2016. In-situ measurements of temperature distribution during transmission laser welding of poly(aryletherketone). AIP Conf. Proc. 1769(1). DOI: 10.1063/1.4963408

36. Alfaro, S. C. A., Vargas, J. A. R., De Carvalho, G. C., and De Souza, G. G. 2015. Characterization of "humping" in the GTA welding process using infrared images. Journal of Materials Processing Technology 223: 216-24. DOI: 10.1016/j.jmatprotec.2015.03.052

37. Cho, C.-H., Hsieh, Y.-C., and Chen, H.-Y. 2015. Welding pool measurement using thermal array sensor. Proc. SPIE 9609, Infrared Sensors, Devices, and Applications V: 960912. DOI: 10.1117/ 12.2187751

38. Zhang, Z., Wen, G., and Chen, S. 2018. Audible sound-based intelligent evaluation for aluminum alloy in robotic pulsed GTAW: Mechanism, feature selection, and defect detection. IEEE Trans. Ind. Informatics 14(7): 2973-2983. DOI: 10.1109/TII.2017.2775218

39. Lv, N., Xu, Y., Li, S., Yu, X., and Chen, S. 2017. Automated control of welding penetration based on audio sensing technology. Journal of Materials Processing Technology 250: 81-98. DOI: 10.1016/j.jmatprotec.2017.07.005

40. Li, P. J., and Zhang, Y. M. Robust sensing of arc length.
2001. IEEE Trans. Instrum. Meas. 50(3): 697-704. DOI: 10.1109/19.930442

41. Li, P. J., and Zhang, Y. M. 2001. Precision sensing of arc length in GTAW based on arc light spectrum. Journal of Manufacturing Science and Engineering 123(1): 62-65. DOI: 10.1115/ 1.1349719

42. Shi, Y. H., Yoo, W. S., and Na, S. J. 2006. Mathematical modelling of rotational arc sensor in GMAW and its applications to seam tracking and endpoint detection. Sci. Tech. Weld. Join. 11(6): 723-730. DOI: $10.1179 / 174329306 \times 153196$

43. Kim, C. H., and Na, S. J. 2001. A study of an arc sensor model for gas metal arc welding with rotating arc part 2: Simulation of an arc sensor in mechanically rotating gas metal arc welding. Proc. Inst. Mech. Eng. Part B: J. Eng. Manuf. 215(9): 1281-1288. DOI: 10.1243/0954405011519213

44. Kotecki, D. J., Cheever, D., and Howden, D. 1972. Mechanism of ripple formation during weld solidification. Welding Journal 51(8): 386-s to 391-s.

45. Renwick, R., and Richardson, R. W. 1983. Experimental investigation of GTA weld pool oscillations. Welding Journal 62(2): 29-s to 35-s.

46. Zacksenhouse, M., and Hardt, D. E. 1983. Weld pool impedance identification for size measurement and control. J. Dyn. Syst. Meas. Control 105(3): 179-184. DOI: 10.1115/1.3140652

47. Xiao, Y. H., and den Ouden, G. 1993. Weld pool oscillation during GTA welding of mild steel. Welding Journal 72(8): 428-s to 434-s.

48. Aendenroomer, A. J. R., and den Ouden, G. 1998. Weld pool oscillation as a tool for penetration sensing during pulsed GTA welding. Welding Journal 77(5): 181-s to 187-s.

49. Andersen, K., and Cook, G. E. 1997. Synchronous weld pool oscillation for monitoring and control. IEEE Trans. Ind. Appl. 33(2): 464-471. DOI: 10.1109/28.568011

50. Lancaster, J. F., ed. 1986. The Physics of Welding, $2^{\text {nd }}$ ed. International Institute of Welding. DOI: 10.1016/C2013-0-03805-4

51. Shi, Y., Li, C., Du, L., Gu, Y., and Zhu, M. 2016. Frequency characteristics of weld pool oscillation in pulsed gas tungsten arc welding. Journal of Manufacturing Processes 24(1): 145-151. DOI: 10.1016/j.jmapro.2016.08.010

52. Hardt, D. E., and Katz, J. M. 1984. Ultrasonic measurement of weld penetration. Welding Journal 63(9): 273-s to 285-s.

53. Fenn, R. 1985. Ultrasonic monitoring and control during arc welding. Welding Journal 64(9): 18-24.

54. Carlson, N. M., and Johnson, J. A. 1988. Ultrasonic sensing of weld pool penetration. Welding Journal 67(11): 239-s to 246-s.

55. Aussel, J. D., Le Brun, A., and Baboux, J. C. 1988. Generating acoustic waves by laser: Theoretical and experimental study of the emission source. Ultrasonics 26(5): 245-255. DOI: 10.1016/ 0041-624X(88)90013-3

56. Graham, G. M., and Ume, I. C. 1997. Automated system for laser ultrasonic sensing of weld penetration. Mechatronics 7(8): 711-721. DOI: 10.1016/s0957-4158(97)00031-7

57. Mi, B., and Ume, C. 2006. Real-time weld penetration depth monitoring with laser ultrasonic sensing system. Journal of Manufacturing Science and Engineering 128(1): 280-286. DOI: 10.1115/ 1.2137747

58. Kita, A. 2005. Measurement of weld penetration depth using non-contact ultrasound method. PhD diss., Georgia Institute of Technology.

59. Dixon, S., Edwards, C., and Palmer, S. B. 1999. Laser-EMAT system for ultrasonic weld inspection. Ultrasonics 37(4): 273-281. DOI: 10.1016/S0041-624X(99)00002-5

60. Chen, W., and Chin, B. A. 1990. Monitoring joint penetration using infrared sensing techniques. Welding Journal 69(4): 181 -s to 185 -s.

61. Wikle, H. C., Kottilingam, S., Zee, R. H., and Chin, B. A. 2001. Infrared sensing techniques for penetration depth control of 
the submerged arc welding process. J. Mater. Process. Technol. 113(1-3): 228-233. DOI: 10.1016/S0924-0136(01)00587-8

62. Nagarajan, S., Banerjee, P., Chen, W. H., and Chin, B. A. 1992. Control of the welding process using infrared sensors. IEEE Trans. Robot. Autom. 8(1): 86-93. DOI: 10.1109/70.127242

63. Alfaro, S. C. A., and Franco, F. D. 2010. Exploring infrared sensoring for real time welding defects monitoring in GTAW. Sensors 10(6): 5962-5974. DOI: 10.3390/s100605962

64. Corlett, B. J., Lucas, J., and Smith, J. S. 1991. Sensors for narrow-gap welding. IEE Proc. A Sci. Meas. Technol. 138(4): 213-222. DOI: 10.1049/ip-a-3.1991.0030

65. Bicknell, A., Smith, J. S., and Lucas, J. 1994. Arc voltage sensor for monitoring of penetration in TIG welds. IEE Proc. Sci. Meas. Technol. 141(6): 513-520. DOI: 10.1049/ip-smt:19941144

66. Bai, P., Wang, Z., Hu, S., Ma, S., and Liang, Y. 2017. Sensing of the weld penetration at the beginning of pulsed gas metal arc welding. Journal of Manufacturing Processes 28(1): 343-350. DOI: 10.1016/j.jmapro.2017.07.002

67. Li, X. R., Shao, Z., Zhang, Y. M., and Kvidahl, L. 2013. Monitoring and control of penetration in GTAW and pipe welding. Welding Journal 92(6): 190-s to 196-s.

68. Zhang, Y. M., Li, X., and Shao, Z. 2017. A method to monitor and control weld penetration in gas tungsten welding and fullposition pipe welding. U.S. patent 9604301.

69. Rokhlin, S. I., and Guu, A. C. 1993. A study of arc force, pool depression, and weld penetration during gas tungsten arc welding. Welding Journal 72(8): 381-s to 390-s.

70. Rokhlin, S. I., and Guu, A. C. 1990. Computerized radiographic sensing and control of an arc welding process. Welding Journal 69(3): 83-s to 97-s.

71. Zhao, D. B., Yi, J. Q., Chen, S. B., Wu, L., and Chen, Q. 2003. Extraction of three-dimensional parameters for weld pool surface in pulsed GTAW with wire filler. Journal of Manufacturing Science and Engineering 125(3): 493-503. DOI: 10.1115/1.1556400

72. Kovacevic, R., and Zhang, Y. M. 1996. Sensing free surface of arc weld pool using specular reflection: Principle and analysis. Proc. Inst. Mech. Eng. Part B: J. Eng. Manuf. 210(6): 553-564. DOI: 10.1243/PIME_PROC_1996_210_154_02

73. Saeed, G., and Zhang, Y. M. 2007. Weld pool surface depth measurement using a calibrated camera and structured light. Measurement Science and Technology 18(8): 2570-2578. DOI: 10.1088/ 0957-0233/18/8/033

74. Song, H., and Zhang, Y. M. 2009. Error analysis of a threedimensional GTA weld pool surface measurement system. Welding Journal 88(7): 141-s to 148-s.

75. Zhang, W., Liu, Y., and Zhang, Y. 2013. Real-time measurement of the weld pool surface in GTAW process. 2013 IEEE Instrum. Meas. Technol. Conf., 1640-1645. Minneapolis, Minn.: IEEE. DOI: 10.1109/I2MTC.2013.6555692

76. Zhang, W., Liu, Y. K., Wang, X., and Zhang, Y. M. 2012. Characterization of three-dimensional weld pool surface in gas tungsten arc welding. Welding Journal 91(7): 195-s to 203-s.

77. Borrelli, F., Bemporad, A., and Morari, M. 2017. Predictive Control for Linear and Hybrid Systems. Cambridge, England: Cambridge University Press. DOI: 10.1017/9781139061759

78. Hartman, D. A., DeLapp, D. R., Cook, G. E., and Barnett, R. J. 1999. Intelligent fusion control throughout varying thermal regions [arc welding]. Conf. Rec. 1999 IEEE Ind. Appl. Conf. ThirtyForth IAS Annu. Meet. (Cat. No.99CH36370) 1: 635-644. Phoenix, Ariz.: IEEE. DOI: 10.1109/IAS.1999.800018

79. Ju, J., Suga, Y., and Ogawa, K. 2002. Penetration control by monitoring molten pool oscillation in TIG arc welding. Int. J. Offshore Polar Eng. 14(2): 145-149.

80. Yudodibroto, B. Y. B., Hermans, M. J. M., Hirata, Y., and den Ouden, G. 2004. Influence of filler wire addition on weld pool oscillation during gas tungsten arc welding. Sci. Technol. Weld. Join. 9(2): 163-168. DOI: 10.1179/136217104225012274
81. Guu, A. C., and Rokhlin, S. I. 1996. Arc weld process control using radiographic sensing. NDT\&E International 29(3): 184. DOI: 10.1016/0963-8695(96)84884-5

82. Yahia, N. B., Belhadj, T., Brag, S., and Zghal, A. 2011. Automatic detection of welding defects using radiography with a neural approach. Procedia Eng. 10: 671-679. DOI: 10.1016/j.proeng.2011. 04.112

83. Song, J.-B., and Hardt, D. E. 1993. Closed-loop control of weld pool depth using a thermally based depth estimator. Welding Journal 72(10): 471-s to 478-s.

84. Boo, K. S., and Cho, H. S. 1994. A self-organizing fuzzy control of weld pool size in GMA welding processes. Control Eng. Pract. 2(6): 1007-1018. DOI: 10.1016/0967-0661(94)91623-3

85. Banerjee, P., Govardhan, S., Wikle, H. C., Liu, J. Y., and Chin, B. A. 1995. Infrared sensing for on-line weld geometry monitoring and control. Journal of Manufacturing Science and Engineering 117(3): 323-330. DOI: 10.1115/1.2804337

86. Vorman, A. R., and Brandt, H. 1976. Feedback control of GTA welding using puddle width measurement. Welding Journal 55(9): 742-749.

87. Ohshima, K., Yamamoto, M., Tanii, T., and Yamane, S. 1992. Digital control of torch position and weld pool in MIG welding using image processing device. IEEE Transactions on Industry Applications 28(3): 607-612. DOI: 10.1109/28.137446

88. Pietrzak, K. A., and Packer, S. M. 1994. Vision-based weld pool width control. Journal of Manufacturing Science and Engineering 116(1): 86-92. DOI: 10.1115/1.2901813

89. Song, J. B., and Hardt, D. E. 1994. Dynamic modeling and adaptive control of the gas metal arc welding process. J. Dyn. Syst. Meas. Control. 116(3): 405-413. DOI: 10.1115/1.2899235

90. Kovacevic, R., and Zhang, Y. M. 1997. Neurofuzzy modelbased weld fusion state estimation. IEEE Control Syst. 17(2): 30-42. DOI: $10.1109 / 37.581293$

91. Zhang, Y. M., and Kovacevic, R. 1998. Neurofuzzy modelbased predictive control of weld fusion zone geometry. IEEE Trans. Fuzzy Syst. 6(3): 389-401. DOI: 10.1109/91.705507

92. Chen, S. B., Zhao, D. B., Wu, L., and Lou, Y. J. 2000. Intelligent methodology for sensing, modeling and control of pulsed GTAW: Part I - Bead-on-plate welding. Welding Journal 79(6): 151-s to 163 -s.

93. Zhao, D. B., Chen, S. B., and Wu, L. 2001. Intelligent control for the shape of the weld pool in pulsed GTAW with filler metal. Welding Journal 80(11): 253-s to 260-s.

94. Tsai, C. H., Hou, K. H., and Chuang, H. T. 2006. Fuzzy control of pulsed GTA welds by using real-time root bead image feedback. Journal of Materials Processing Technology 176(1-3): 158-67. DOI: 10.1016/j.jmatprotec.2006.02.027

95. Kong, M., and Chen, S. 2009. Al alloy weld pool control of welding robot with passive vision. Sensor Review 29(1): 28-37. DOI: 10.1108/02602280910926733

96. Chen, H., Lv, F., Lin, T., and Chen, S. 2009. Closed-loop control of robotic arc welding system with full-penetration monitoring. J. Intell. Robot. Syst. 56: 565-578. DOI: 10.1007/s10846-0099329-7

97. Liu, Y. K., and Zhang, Y. M. 2014. Model-based predictive control of weld penetration in gas tungsten arc welding. IEEE Trans. Control Syst. Technol. 22(3): 955-966. DOI: 10.1109/TCST. 2013.2266662

98. Liu, Y. K., Zhang, W. J., and Zhang, Y. M. 2015. Nonlinear modeling for 3D weld pool characteristic parameters in GTAW. Welding Journal 94(7): 231-s to 240-s.

99. Liu, Y. K., and Zhang, Y. M. 2013. Control of 3D weld pool surface. Control Eng. Pract. 21(11): 1469-1480. DOI: 10.1016/j. conengprac.2013.06.019

100. Liu, Y., and Zhang, Y. 2015. Iterative local ANFIS-based human welder intelligence modeling and control in pipe GTAW process: A data-driven approach. IEEE/ASME Trans. Mechatronics 
20(3): 1079-1088. DOI: 10.1109/TMECH.2014.2363050

101. Liu, Y. K., and Zhang, Y. M. 2017. Supervised learning of human welder behaviors for intelligent robotic welding. IEEE Trans. Autom. Sci. Eng. 14(3): 1532-1541. DOI: 10.1109/TASE. 2015.2453351

102. Liu, Y. K., Zhang, Y. M., and Kvidahl, L. 2014. Skilled human welder intelligence modeling and control: Part I - Modeling. Welding Journal 93(2): 46-s to 52-s.

103. Liu, Y. K., Zhang, Y. M., and Kvidahl, L. 2014. Skilled human welder intelligence modeling and control: Part II - Analysis and control applications. Welding Journal 93(5): 162-s to 170-s.

104. Liu, Y. K., and Zhang, Y. M. 2015. Controlling 3D weld pool surface by adjusting welding speed. Welding Journal 94(4): 125-s to 134-s.

105. Liu, Y. K., and Zhang, Y. M. 2014. Control of human arm movement in machine-human cooperative welding process. Control Eng. Pract. 32: 161-171. DOI: 10.1016/j.conengprac.2014.08.003

106. Liu, Y. K., and Zhang, Y. M. 2017. Fusing machine algorithm with welder intelligence for adaptive welding robots. Journal of Manufacturing Processes 27: 18-25. DOI: 10.1016/j.jmapro. 2017.03.015

107. Liu, Y., and Zhang, Y. 2019. Human welder 3-D hand movement learning in virtualized GTAW: Theory and experiments. Trans. Intell. Weld. Manuf. 2(1): 3-25. DOI: 10.1007/978-981-108740-0_1

108. Traidia, A., and Roger, F. 2011. Numerical and experimental study of arc and weld pool behaviour for pulsed current GTA welding. Int. J. Heat Mass Transf. 54: 2163-2179. DOI: 10.1016/ j.ijheatmasstransfer.2010.12.005

109. Mougenot, J., Gonzalez, J. J., Freton, P., and Masquère, M. 2013. Plasma-weld pool interaction in tungsten inert-gas configuration. J. Phys. D: Appl. Phys.: 46. DOI: 10.1088/0022-3727/46/13/ 135206

110. Lecun, Y., Bengio, Y., and Hinton, G. 2015. Deep learning. Nature 521: 436-444. DOI: 10.1038/nature14539

111. Samuel, A. L. 1959. Some studies in machine learning using the game of checkers. IBM J. Res. Dev. 3: 210-229. DOI:

$10.1147 / \mathrm{rd} .33 .0210$

112. Mitchell, T. 1997. Machine Learning, $1^{\text {st }}$ ed. McGraw Hill Higher Education.

113. Andersen, K., Cook, G. E., Karsai, G., and Ramaswamy, K. 1990. Artificial neural networks applied to arc welding process modeling and control. IEEE Trans. Ind. Appl. 26(5): 824-830. DOI: 10.1109/28.60056

114. Baraka, A., Panoutsos, G., and Cater, S. 2015. A real-time quality monitoring framework for steel friction stir welding using computational intelligence. J. Manuf. Processes 20(1): 137-148. DOI: 10.1016/j.jmapro.2015.09.001

115. Kim, I. S., Son, J. S., Park, C. E., Lee, C. W., and Prasad, Y. K. D. V. 2002. A study on prediction of bead height in robotic arc welding using a neural network. J. Mater. Process. Technol. 130-131: 229-234. DOI: 10.1016/S0924-0136(02)00803-8

116. Kim, I. S., Son, J. S., Lee, S. H., and Yarlagadda P. K. D. V. 2004. Optimal design of neural networks for control in robotic arc welding. Robot. Comput. Integr. Manuf. 20(1): 57-63. DOI: 10.1016/S0736-5845(03)00068-1

117. Yuguang, Z., Kai, X., and Dongyan, S. 2013. An improved artificial neural network for laser welding parameter selection and prediction. Int. J. Adv. Manuf. Technol. 68: 755-762. DOI: 10.1007/ s00170-013-4796-1

118. Acherjee, B., Mondal, S., Tudu, B., and Misra, D. 2011. Application of artificial neural network for predicting weld quality in laser transmission welding of thermoplastics. Appl. Soft Comput. 11(2): 2548-2555. DOI: 10.1016/j.asoc.2010.10.005

119. Gupta, S. K., Pandey, K. N., and Kumar, R. 2018. Artificial intelligence-based modelling and multi-objective optimization of friction stir welding of dissimilar AA5083-O and AA6063-T6 aluminium alloys. Proc. Inst. Mech. Eng., Part L: J. Mater. Des. Appl. 232(4): 333-342. DOI: 10.1177/1464420715627293

120. Okuyucu, H., Kurt, A., and Arcaklioglu, E. 2007. Artificial neural network application to the friction stir welding of aluminum plates. Mater. Des. 28(1): 78-84. DOI: 10.1016/j.matdes.2005.06. 003

121. Zhao, D., Ren, D., Zhao, K., Pan, S., and Guo, X. 2017. Effect of welding parameters on tensile strength of ultrasonic spot welded joints of aluminum to steel - By experimentation and artificial neural network. J. Manuf. Processes 30: 63-74. DOI: 10.1016/ j.jmapro.2017.08.009

122. Zhao, D., Zhao, K., Ren, D., and Guo, X. 2017. Ultrasonic welding of magnesium-titanium dissimilar metals: A study on influences of welding parameters on mechanical property by experimentation and artificial neural network. Journal of Manufacturing Science and Engineering 139(3): 031019. DOI: 10.1115/1.4035539

123. Manikya Kanti, K., and Srinivasa Rao, P. 2008. Prediction of bead geometry in pulsed GMA welding using back propagation neural network. J. Mater. Process. Technol. 200(1-3): 300-305. DOI: 10.1016/j.jmatprotec.2007.09.034

124. Mirapeix, J., García-Allende, P. B., Cobo, A., Conde, O. M., and López-Higuera, J. M. 2007. Real-time arc-welding defect detection and classification with principal component analysis and artificial neural networks. NDT\&E Int. 40(4): 315-323. DOI: 10.1016/ j.ndteint.2006.12.001

125. Valavanis, I., and Kosmopoulos, D. 2010. Multiclass defect detection and classification in weld radiographic images using geometric and texture features. Expert Syst. Appl. 37(12): 7606-7614. DOI: 10.1016/j.eswa.2010.04.082

126. Hou, W., Wei, Y., Guo, J., Jin., Y., and Zhu, C. 2018. Automatic detection of welding defects using deep neural network. $J$. Phys.: Conf. Ser. 933: 012006. DOI: 10.1088/1742-6596/933/1/ 012006

127. Sassi, P., Tripicchio, P., and Avizzano, C. A. 2019. A smart monitoring system for automatic welding defect detection. IEEE Trans. Ind. Electron 66(12): 9641-9650. DOI: 10.1109/TIE.2019. 2896165

128. You, D., Gao, X., and Katayama, S. 2014. Multisensor fusion system for monitoring high-power disk laser welding using support vector machine. IEEE Trans. Ind. Informatics 10(2): 1285-1295. DOI: 10.1109/TII.2014.2309482

129. You, D., Gao, X., and Katayama, S. 2015. WPD-PCA-based laser welding process monitoring and defects diagnosis by using FNN and SVM. IEEE Trans. Ind. Electron. 62(1): 628-636. DOI: 10.1109/TIE.2014.2319216

130. Zhang, Y., You, D., Gao, X., Zhang, N., and Gao, P. P. 2019. Welding defects detection based on deep learning with multiple optical sensors during disk laser welding of thick plates. J. Manuf. Syst. 51: 87-94. DOI: 10.1016/j.jmsy.2019.02.004

131. Cruz, J. G., Torres, E. M., and Absi Alfaro, S. C. 2015. A methodology for modeling and control of weld bead width in the GMAW process. J. Brazilian. Soc. Mech. Sci. Eng. 37: 1529-1541. DOI: $10.1007 / \mathrm{s} 40430-014-0299-8$

132. Günther, J., Pilarski, P. M., Helfrich, G., Shen, H., and Diepold, K. 2016. Intelligent laser welding through representation, prediction, and control learning: An architecture with deep neural networks and reinforcement learning. Mechatronics 34: 1-11. DOI: 10.1016/j.mechatronics.2015.09.004

133. Jin, Z., Li, H., and Gao, H. 2019. An intelligent weld control strategy based on reinforcement learning approach. Int. J. Adv. Manuf. Technol. 100: 2163-2175. DOI: 10.1007/s00170-018-2864-2

134. Li, C., Wang, Q. Y., Jiao, W. H., Johnson, M., and Zhang, Y. M. 2020. Deep learning-based detection of penetration from weld pool reflection images. Welding Journal 99(9): 239-s to 245-s. DOI: 10.29391/2020.99.022

135. Pires, J. N., Loureiro, A., Godinho, T., Ferreira, P., Fernando, B., and Morgado, J. 2003. Welding robots. IEEE Robot. Autom. 
Mag. 10(2): 45-55. DOI: 10.1109/MRA.2003.1213616

136. Sprovieri, J. 2016. New technology for robotic welding. assemblymag.com/articles/93555-new-technology-for-robotic-welding

137. Pan, Z., Polden, J., Larkin, N., Van Duin, S., and Norrish, J. 2012. Recent progress on programming methods for industrial robots. Robot. Comput. Integr. Manuf. 28(2): 87-94. DOI: 10.1016/ j.rcim.2011.08.004

138. Zhang, W., and Zhang, Y. M. 2012. Modeling of human welder response to 3D weld pool surface: Part I — Principles. Welding Journal 91(11): 310-s to 318-s.

139. Zhang, W., and Zhang, Y. 2012. Modeling of human welder response to 3D weld pool surface: Part II - Results and analysis. Welding Journal 91(11): 329-s to 337-s.

140. Liu, Y., Zhang, W., and Zhang, Y. 2015. Dynamic neurofuzzy-based human intelligence modeling and control in GTAW. IEEE Trans. Autom. Sci. Eng. 12(1): 324-335. DOI: 10.1109/TASE. 2013.2279157

141. Zhang, L., Gao, H., Zhang, G., and Wu, L. 2006. Agentbased modelling and control of tele-robotic welding system. Proc. Inst. Mech. Eng. Part B: J. Eng. Manuf. 220(12): 2087-2094. DOI: 10.1243/09544054JEM544

142. Ni, D., Yew, A. W. W., Ong, S. K., and Nee, A. Y. C. 2017. Haptic and visual augmented reality interface for programming welding robots. Adv. Manuf. 5: 191-198. DOI: 10.1007/s40436017-0184-7

143. Liu, Y., and Zhang, Y. 2015. Toward welding robot with human knowledge: A remotely-controlled approach. IEEE Trans. Au- tom. Sci. Eng. 12: 769-774. DOI: 10.1109/TASE.2014.2359006

144. Wang, Q., Jiao, W., Yu, R., Johnson, M. T., and Zhang, Y. 2019. Modeling of human welders' operations in virtual reality human-robot interaction. IEEE Robot. Autom. Lett. 4: 2958-2964. DOI: 10.1109/LRA.2019.2921928

145. Wang, Q., Cheng, Y., Jiao, W., Johnson, M. T., and Zhang, Y. M. 2019. Virtual reality human-robot collaborative welding: A case study of weaving gas tungsten arc welding. J. Manuf. Processes. 48: 210-217. DOI: 10.1016/j.jmapro.2019.10.016

146. Erden, M. S., and Billard, A. 2016. Robotic assistance by impedance compensation for hand movements while manual welding. IEEE Trans. Cybern. 46(11): 2459-2472. DOI: 10.1109/TCYB.2015. 2478656

147. Wang, Q., Jiao, W., Yu, R., Johnson, M. T., and Zhang, Y. M. 2020. Virtual reality robot-assisted welding based on human intention recognition. IEEE Trans. Autom. Sci. Eng. 17(2): 799-808. DOI: 10.1109/TASE.2019.2945607

YUMING ZHANG (yuming.zhang@uky.edu) and QIYUE WANG are with the Institute for Sustainable Manufacturing and Department of Electrical and Computer Engineering, University of Kentucky, Lexington, Ky. YUKANG LIU is with Cognex Corp., Natick, Mass.

\section{Welding Journal Now Publishing Direct Object Identifier (DOI) Numbers}

Dear members of the welding research community,

Note that in each issue of the Welding Journal Research Supplement, we are including Direct Object Identifier (DOI) numbers with each of the papers published in print and online. A direct object identifier is a unique alphanumeric string assigned by a registration agency (we are using Crossref.org) to identify content and provide a persistent link to its location on the Internet. Our decision to begin assigning a DOl for each paper comes directly from a request by the research community.

As part of our obligation to Crossref.org, we are asked to provide DOl numbers, when available, in the references section of papers. So, if you have submitted a paper to the Welding Journal or are planning on submitting a paper, we ask that you update your references to include DOI numbers whenever possible.

Thank you. 